\title{
Facial mimicry and metacognitive judgments in emotion recognition - modulated by social anxiety and autistic traits?
}

\author{
Julia Folz ${ }^{1,2}$, Rüya Akda $\breve{g}^{3}$, Milica Nikolić ${ }^{1,2,4}$, Henk van Steenbergen ${ }^{1,2}$, Mariska E. Kret ${ }^{1,2}$ \\ ${ }^{1}$ Department of Cognitive Psychology, Institute of Psychology, Leiden University, \\ ${ }^{2}$ Leiden Institute for Brain and Cognition (LIBC), Leiden University \\ ${ }^{3}$ Department of Developmental and Educational Psychology, Institute of Psychology, Leiden University \\ ${ }^{4}$ Department of Developmental Psychology, University of Amsterdam
}

*Corresponding author: j.folz@fsw.leidenuniv.nl (J. Folz)

ORCID identifiers: 0000-0002-7572-8528 (J.F.), 0000-0002-7898-6012 (M.N.), 0000-0003-1917-6412 (H.v.S.), 0000-0002-3197-5084 (M.E.K.) 


\begin{abstract}
Both individuals with social anxiety disorder and individuals with autism spectrum disorder show alterations in the perception of others' emotional facial expressions. Additionally, mimicry of an observed expression as well as the assessment of one's own performance, i.e. metacognition, may be altered in these individuals. Using a non-clinical sample ( $\mathrm{N}=57)$, we examined whether emotion recognition is linked to facial mimicry and confidence in one's performance, as well as potential alterations in this link associated with social anxiety and autistic traits. While participants were presented with videos of spontaneous emotional facial expressions, we measured their facial muscle activity, and asked them to label the expressions and indicate their confidence levels in accurately labelling the expressions. Our results showed that confidence in emotion recognition was lower with higher social anxiety trait levels even though actual recognition was not related to social anxiety trait levels. Higher autistic trait levels, in contrast, were associated with worse recognition as well as a weakened link between performance and facial mimicry. Consequently, social anxiety might not affect emotion recognition itself but the top-down evaluation in an emotion recognition context, whereas individuals with autism may integrate information promoting emotion recognition, i.e. sensorimotor simulations, to a lesser degree.
\end{abstract}

Keywords: Autistic Traits, Social Anxiety, Facial Affect Recognition, Mimicry, Metacognition 


\section{Introduction}

The expression "her face says it all" exemplifies the fundamental contribution of nonverbal signals and cues in the communication of inner states (Frith, 2009). According to social-functional approaches, emotional expressions are crucial to guide social interactions by informing about others' states, evoking coordinated emotional responses, and incentivizing social behavior (Keltner \& Kring, 1998). An atypical processing of others' emotions has been associated with various psychiatric disorders, such as social anxiety disorder (SAD) and autism spectrum disorder (ASD). Research in both clinical populations has revealed a link between alterations in processing other's emotional expressions and dysfunctional social behavior (Boraston et al., 2008; Gilboa-Schechtman \& ShacharLavie, 2013; Gutiérrez-García \& Calvo, 2016; Philip et al., 2010; Trevisan \& Birmingham, 2016). Crucially, studies on facial emotion perception have predominantly used static pictures of exaggeratedly posed expressions as stimuli, making it difficult to translate the findings to real-life situations with spontaneous emotion displays, which are characterized by their richness in the dynamics, ambiguity and subtlety (Ambadar et al., 2005; Cohn, 2007; Dobs et al., 2018; Krumhuber et al., 2013).

The accurate identification of an observed emotional expression is a key component in the interpretation of an expresser's emotional state, yet links between emotion recognition and other processes in emotion perception are still not well described. Facial mimicry, i.e. the mirroring of an observed expression, is one process that has been suggested to promote the recognition of others' emotions, (e.g. Drimalla et al., 2019; Künecke et al., 2014). In contrast to this bottom-up information channel, the top-down assessment of one's recognition performance, i.e. metacognition, might also provide relevant feedback about emotion processing (Kelly \& Metcalfe, 2011). Importantly, alterations in both facial mimicry and metacognitive judgments have been described in a wide range of psychiatric symptoms, including those related to ASD and SAD (Davies et al., 2016; Rouault et al., 2018). The current study used a non-clinical sample with varying levels of autistic and social anxiety traits to examine the link between emotion recognition and these two different processes, i.e. facial 
mimicry and confidence in emotion recognition abilities, as well as their potential alterations associated with these traits.

\subsection{General emotion recognition alterations in SAD and ASD}

While emotion recognition deficits in SAD have sporadically been reported in the past (e.g. Montagne et al., 2006), most research did not find alterations (Bui et al., 2017) or even a higher sensitivity to emotional expressions (Arrais et al., 2010; Joormann \& Gotlib, 2006). Heightened attention to social cues also stands at the basis of established theoretical models on social anxiety (Rapee \& Heimberg, 1997, Clark \& Wells, 1995) and has predominantly received support in form of a negativity bias (e.g. Amin et al., 1998; Hirsch \& Clark, 2004; Machado-de-Sousa et al., 2010). Correspondingly, healthy individuals with high social anxiety trait levels showed an emotion recognition advantage (Hunter et al., 2009), and specifically for negative expressions in past studies (Gutiérrez-García \& Calvo, 2017; Richards et al., 2002) . Individuals with an ASD diagnosis, in contrast, have been found to have an overall deficit in recognizing emotional facial expressions, with the most pronounced impairment for fearful expressions (Frank et al., 2018; Sucksmith et al., 2013; Uljarevic \& Hamilton, 2013). Interestingly, a recent study investigating the link between autistic trait levels in healthy individuals and facial emotion recognition reported an improved recognition of sad expressions, but a worse recognition of angry expressions, with higher trait levels (Green \& Guo, 2018). Despite some inconsistencies in the literature, it seems that social anxiety and autism are related quite distinctly to facial emotion recognition, with only the latter showing clear deficits. Importantly, observed alterations in emotion recognition in a laboratory setting also show links to difficulties in navigating in the 'real' social world. For example, everyday-life adaptive functioning has been found to be positively related to emotion recognition performance in individuals with ASD (Trevisan \& Birmingham, 2016). An understanding of systematic alterations in emotion recognition associated with autism and social anxiety might therefore be necessary to improve day-to-day experiences. 


\subsection{Emotional expressions in the real world}

Even though humans are confronted with a broad variety of rich emotional facial expressions in daily life, research on emotion recognition has mostly employed static images of exaggerated and posed stimuli (Kret et al., 2020). In order to gain more insights into the actual processing of our environment, several calls for more naturalistic stimuli have been raised, thus increasing ecological validity of the findings in the lab (Kret, 2015; Sonkusare et al., 2019). Accordingly, novel data sets with more naturalistic emotional expressions, varying in both the degree as well as specific facets, have become more popular in the last years (Dobs et al., 2018; Krumhuber et al., 2017). In the case of facial displays of emotion, the dynamics of an expression play a crucial role in the way they are processed. Stronger responses to dynamic stimuli have been described on multiple levels, including subjective experience (Krumhuber et al., 2013), physiology (Rymarczyk et al., 2011), as well as neuronal responses (Schultz \& Pilz, 2009). Whether naturalistic facial expressions are also better recognized is, however, debated (for: Alves, 2013; against: Krumhuber et al., 2019), and results seem to strongly depend on the specific stimulus set (Sauter \& Fischer, 2018). Nevertheless, especially for more subtle and ambiguous displays, a dynamic stimulus with a change in the expression has been found to promote recognition (Ambadar et al., 2005; Krumhuber et al., 2013). As those displays are most common in daily life, naturalistic emotional expressions might also provide more valid insights into emotion processing alterations in clinical populations. When comparing the recognition of static and dynamic emotion displays, emotion recognition deficits in ASD were not generally affected by the presence of dynamic information, but dynamic sad expressions were less well recognized than static ones (Enticott et al., 2014). Further, individuals with high levels of social anxiety only showed an advantage in recognizing static but not dynamic expressions of anger (Torro-Alves et al., 2016). Hence, paradigms involving static expressions might provide a distorted estimate of real-life emotion recognition in clinical populations. 


\subsection{Facial mimicry and its alterations in SAD and ASD}

When observing an individual expressing an affective state via the face, an automatic imitation of the observed facial expression, i.e. facial mimicry, has been consistently described in humans (Dimberg, 1982). Most studies employing facial electromyography (fEMG) to capture facial mimicry have focused on measuring activity over two muscle regions, the Zygomaticus Major and the Corrugator Supercilii (for simplicity referred to as "zygomaticus" and "corrugator" in the following). The associations which were initially made between activity of these muscle regions and the experience of distinct emotional states (Ekman \& Rosenberg, 2005) were also consistently reported in response to dynamic emotional displays: Strongest evidence has been found for an increase in zygomaticus activity when happy facial expressions were viewed which was interpreted as mimicry of the smile (compared to neutral/baseline: Künecke et al., 2014; Sato et al., 2008; Rymarczyk et al., 2011, 2016a; compared to angry: Dijk et al., 2018; contrasted with other muscles: Hess \& Blairy, 2001; Olszanowski et al., 2020). Displays of happiness were also responded to with a decrease in corrugator activity in these studies, which could be an indication of relaxation linked to positive affect. Moreover, most facial mimicry studies could confirm the proposed link between enhanced corrugator activity and anger (e.g. Künecke et al., 2014; Peter-Ruf et al., 2017; Sato et al., 2008; compared to happy: Dijk et al., 2018; compared to other muscles: Hess \& Blairy, 2001; Olszanowski et al., 2020), with only sporadic reports of little (Rymarczyk et al., 2011) or no evidence (Rymarczyk et al., 2016). Lastly, in line with the EMFACS mapping, the few studies on mimicry of sad expressions supported a suggested corrugator activity increase, with the effect being less pronounced compared to displays of anger (Hess \& Blairy, 2001; Künecke et al., 2014; Olszanowski et al., 2020). Studies investigating the relationship between social anxiety and facial mimicry have reported inconsistent results: while some studies found intact mimicry in non-clinical but high socially anxious individuals (Dijk et al., 2018; Peter-Ruf et al., 2017), others demonstrated enhanced mimicry of negative expressions and diminished mimicry of positive ones (Dimberg, 1997; Vrana \& Gross, 2004) or stronger differential muscle activity between happy and angry expressions, for both the zygomaticus and the corrugator (Dimberg $\&$ Thunberg, 2007). The literature on the link between autism and facial mimicry is less controversial. Reduced automatic mimicry in autistic individuals has been consistently reported (Davies et al., 2016). 
Importantly, altered mimicry in ASD could not be explained by a generally lower facial expressiveness in people with ASD but by a mismatch between observed and produced facial muscle activity patterns (McIntosh et al., 2006; Rozga et al., 2013; Weiss et al., 2003). Further, when instructed to imitate, individuals with an ASD diagnosis (McIntosh et al., 2006) or elevated autistic trait levels (Hermans et al., 2009) did show mimicry of all facial expressions. Thus, despite having the ability to mimic, facial mimicry does not seem to automatically occur in individuals with ASD. Taken together, while there is no strong evidence for altered mimicry of all facial expressions in SAD, individuals with ASD seem to show less automatic mimicry.

\subsection{Altered mimicry as a mechanism of altered emotion recognition}

Instead of being only an epiphenomenon, facial mimicry has been claimed to have a function in emotion perception, namely as mechanism to promote emotion recognition (Drimalla et al., 2019; Künecke et al., 2014). Peripheral signals have been proposed to serve as an important information source in many seminal theories on emotion (Damasio, 1996,; James, 1884). Within this theoretical framework, facial muscle activity has specifically been suggested to determine the elicitation of distinct emotional states (e.g. Ekman et al., 1983; Oberman et al., 2007), as suggested by the "facial feedback hypothesis" (Buck, 1980). Accordingly, specific facial muscle activations could be associated to the experience of specific emotions (Brown \& Schwartz, 1980). Not only the direct experience of an emotion but also the observation of another individual expressing an emotion via the face has been linked to the elicitation of corresponding facial muscle activation patterns (Dimberg, 1982). This finding is in line with the idea that humans automatically share observed affective states, including their associated physiological signature, which has been coined "emotional contagion" (Hatfield et al., 1993; Prochazkova \& Kret, 2017). From a functional perspective, the embodiment of another person's emotion could provide insights into their mental states, i.e. mirrored bodily states could serve as feedback about the other (Niedenthal, 2007). This view was supported by studies that showed a decline in emotion recognition performance if facial mimicry was voluntarily (Stel \& van Knippenberg, 2008) or artificially (Neal \& Chartrand, 2011) blocked. However, facial mimicry does not seem to be necessary for facial emotion recognition as demonstrated in patients with the Möbius 
syndrome (Bogart \& Matsumoto, 2010). The potential function of facial mimicry in emotion recognition has recently been evaluated in a meta-analysis which could not provide evidence for a significant relationship (Holland et al., 2020). One confounding factor might, once again, be the ecological validity of the emotional expressions. For example, path analyses showed that the positive relationship between the experience of an emotion, which was predicted by facial mimicry, and emotion recognition was specifically pronounced when dynamic stimuli were presented (Sato et al., 2008). Further, the strength of the putative link between facial mimicry and emotion recognition might vary across individuals. Given the above-mentioned atypical patterns in emotion recognition, a closer examination of potential variations in this link should be especially fruitful in clinical populations. While past research on facial mimicry in individuals with ASD and SAD has focused on describing alterations in the process itself, differences in the function of mimicry are still unclear. Therefore, our study aimed to examine the role of social anxiety traits and autistic traits in the relationship between facial mimicry and emotion recognition. This knowledge should not only advance a fundamental understanding of social anxiety and autism but might also provide implications for clinical research and treatment.

\subsection{Altered metacognition in emotion recognition}

An impairment in metacognitive abilities, i.e. misperceptions of one's own performance, has been proposed as a shared characteristic in different psychiatric disorders (Nordahl et al., 2019; Rouault et al., 2018). Despite being an immanent feature of human social interactions (Frith, 2012), metacognition in the domain of emotion recognition is scarcely researched in healthy individuals (however see: Bègue et al., 2019; Dentakos et al., 2019; Kelly \& Metcalfe, 2011). According to the few available studies on emotion recognition in healthy adults, a reliable metacognitive resolution, i.e. a clear subjective discrimination between correct and incorrect responses, next to a general overconfidence has been found (Bègue et al., 2019; Dentakos et al., 2019). Further, only direct trialby-trial ratings, i.e. 'relative meta-accuracy', and not global beliefs about one's abilities were found to be predictive of performance in emotion recognition tasks (Kelly \& Metcalfe, 2011). Theoretical accounts on the development and maintenance of social anxiety have highlighted the importance of a 
negatively biased view on one's own performance in a social context (Rapee \& Heimberg, 1997; Clark $\&$ Wells, 1995), together with an excessive monitoring of the self (Hartman, 1983). This global pessimistic judgment of one's own abilities might have evolved via repeated underestimation of (social) abilities (Müller-Pinzler et al., 2019). Metacognitive abilities in emotion recognition have, however, not been directly tested in individuals with SAD yet. Considering ASD, while some studies on confidence judgments within an emotion recognition context reported no differences between adults with ASD and controls (Sawyer et al., 2014; Wang \& Adolphs, 2017), a more recent study challenged these findings. More specifically, evidence for a decreased metacognitive sensitivity, i.e. both over- and underconfidence in social cognitive tasks including emotion recognition, but not in general cognitive tasks, in patients with ASD has been found (DeBrabander et al., 2020). Moreover, in an assessment of one's own interpersonal skills, adolescents with ASD were found to be overconfident compared to their parents' judgments (Locke \& Mitchell, 2016). Thus, opposed to individuals with SAD, few studies on metacognitive judgments of social cognition in individuals with ASD do not suggest a generalized underconfidence but, rather, a more complex pattern of alteration. Given the limited knowledge about metacognition in the domain of emotion recognition and its relation to autism and social anxiety, the current study aimed to explore two assumptions: (1) whether the negatively biased assessment of one's performance in social situations in people with high social anxiety trait levels also translates to emotion recognition, and (2) whether the decreased metacognitive sensitivity related to higher autistic traits in the social-cognitive domain also specifically holds for emotion recognition performance. Given that first attempts of implementing Metacognitive Training (MCT; Wells, 2009) in interventions targeting individuals with SAD (Nordahl \& Wells, 2018) and ASD (Goodman et al., 2017) seem promising, our study could provide support for its potential usefulness in the domain of emotion recognition.

\subsection{Objectives of the current study}

Based on previous research hinting towards a putative role of facial mimicry in emotion recognition alterations as well as altered metacognitive judgments in social skill assessment in individuals with SAD and ASD, we examined whether social anxiety and autistic traits modulate the 
links between facial mimicry and emotion recognition as well as between confidence judgments and emotion recognition. To investigate this, our participants first passively viewed naturalistic video clips of different facial expressions of emotion while we measured their facial muscle activity. In a subsequent task, participants indicated how strongly they associated the expressions with distinct emotion categories and were asked how confident they were in their judgments. This approach allowed us to measure not only their emotion recognition ability but also automatic facial mimicry to the displayed expressions and their metacognitive judgments (confidence in their ability to recognize emotions accurately). Moreover, they completed two questionnaires assessing autistic traits and social anxiety traits which enabled us to investigate the extent to which variability in the links between these constructs could be explained by social anxiety and autistic traits in our healthy sample. Despite sharing social interaction difficulties in the global disorder definitions, previous research on emotion recognition, facial mimicry and metacognitive judgments showed different alterations in the two clinical populations. Therefore, we also expected to find distinct relationships between these constructs for the two trait dimensions.

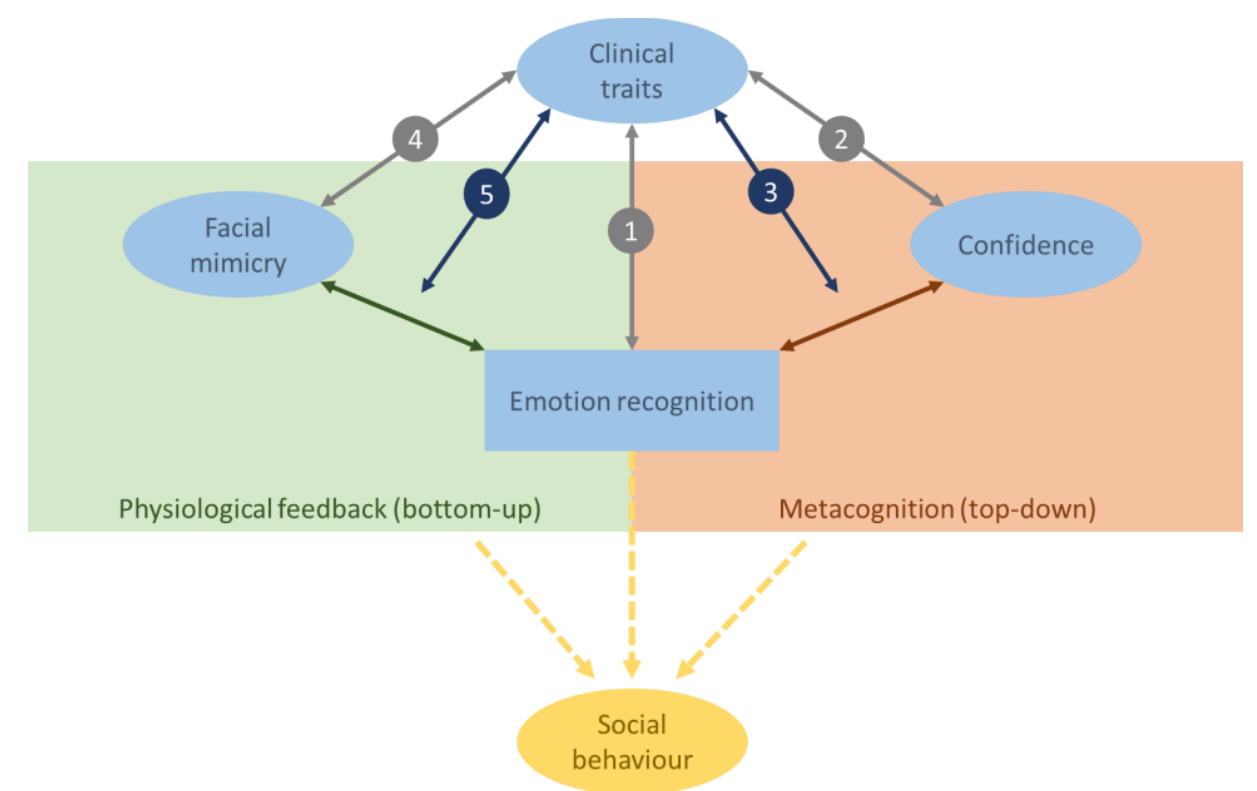

Fig. 1. Visualization of the investigated relationships within this study. Arrows are numbered according to the analysis references in the text, with the grey-coloured arrows representing commonly researched relationships and the blue-coloured arrows representing novel approaches. Yellow components in the figure are not subject of this research but highlight potential implications. 
More specifically, confirming the suggested heightened sensitivity to social cues, higher levels of social anxiety were expected to be related to a recognition advantage of facial expressions, i.e. higher accuracy rates (Analysis 1 in Fig. 1). According to the negativity bias findings, this advantage should be specifically pronounced for negative expressions, i.e. anger, fear and sadness. As socially anxious individuals have been found to report a generalized underconfidence in their social skills, we expected that, despite an improved emotion recognition performance, elevated social anxiety traits would be related to reduced overall confidence in the performance (Analysis 2 in Fig. 1). Moreover, based on observations that confidence judgments were predictive of emotion recognition accuracy in healthy subjects, we would like to explore whether the scaling of confidence judgments to actual emotion recognition performance is altered depending on SA traits (Analysis 3 in Fig. 1). In line with a proposed facilitating role of facial mimicry in emotion recognition, stronger facial mimicry might be assumed with higher social anxiety traits (Analysis 4 in Fig. 1). Empirical evidence for both relationships, i.e. social anxiety and facial mimicry as well as facial mimicry and emotion recognition, is, however, inconclusive. Therefore, we aimed to directly test whether the role of facial mimicry in emotion recognition is altered depending on an individual's social anxiety traits (Analysis 5 in Fig. 1).

Regarding autistic traits, we expected to observe an overall worse recognition of naturalistic dynamic facial expressions with higher levels of autistic traits, which should be most pronounced for fearful faces (Analysis 1 in Fig. 1). Higher autistic trait levels were further expected to be associated with less facial mimicry, and this reduction was expected to be strongest for negative expressions (Analysis 4 in Fig. 1). Further, as automatic facial mimicry has been suggested to be impaired in ASD, the information about facial muscle activity might also be less well integrated in emotion recognition traits (Analysis 5 in Fig. 1). Accordingly, we explored whether a positive relationship between facial mimicry and emotion recognition would be less pronounced with higher autistic trait levels. Lastly, extending on the few findings in clinical samples, we expected a decrease in metacognitive sensitivity with higher autistic traits (Analysis 3 in Fig. 1). Hence, confidence judgments should be less predictive of actual emotion recognition accuracy in individuals with higher autistic trait levels. Given the little 
and inconclusive evidence on metacognition in emotion recognition in healthy and clinical populations, our analyses regarding this research question were explorative.

2. Method

\subsection{Participants}

Fifty-seven healthy participants were recruited from the Leiden University student population (50 female and seven male). Their ages ranged from 18 to 30 years old $(M=22.75, S D=3.27)$ and they all reported normal or corrected-to-normal vision. None of the participants reported current or past psychological or neurological disorders. Participation in the study was voluntary and written consent was obtained prior to the experiment. Participants received either two university credits or a monetary reward of six euros as reimbursement. The study has been executed in accordance with the Declaration of Helsinki and approved by the local ethics committee of the Faculty of Social and Behavioral Sciences at Leiden University (\# 2020-02-10-M.E. Kret-V1-2117).

In the scope of a Master thesis project, an a priori power analysis was run for this study, treating clinical traits as categorical variable (low vs. high trait levels). Based on a similar previous study which found significant group effects with medium effect sizes (Zwick \& Wolkenstein, 2017), we estimated our ideal sample size with the Power Analysis for General ANOVA application (PANGEA; Westfall, 2015). With 30 participants per group, i.e. 60 participants in total, a group effect of $d=.50$ should be found with a power of .901 . Because of the COVID-19 regulations in the Netherlands, we had to stop data collection prematurely and ended up with 57 participants in total. For the analyses in this manuscript, we treated the clinical trait dimensions as continuous variables, thereby increasing the validity of the approach as well as statistical power (Altman \& Royston, 2006).

\subsection{Stimuli}

Following the call for more naturalistic stimuli in research on emotion perception, we chose the FEEDTUM database (Wallhoff et al., 2006) as a source for our stimuli. This database encompasses videotaped spontaneous (i.e. non-instructed) reactions to video clips inducing the six different basic 
emotions and neutral control expressions. Similar to a previous study investigating facial mimicry and emotion recognition in depression (Zwick \& Wolkenstein, 2017), we included facial expressions of anger, fear, happiness, sadness, surprise, and neutral. For each facial expression, video clips of ten individuals (five females and five males) were selected based on (1) the quality of the video clips, i.e. no blur or movement artifacts, and (2) the presence of a clear facial expression of emotion in each video clip (visual inspection). All selected video clips were evaluated by the automated facial expressions recognition software FaceReader 7.1 (Noldus, 2014) to ensure that the emotion label of the stimulus provided by the database was expressed in the video. After identifying the most suitable video clip for each identity and each expression category, the video clips were cut to a uniform length of 2 seconds ( $500 \mathrm{~ms}$ neural expression followed by $1500 \mathrm{~ms}$ of each category's expression). Lastly, the video clips were standardized by removing the original backgrounds using Adobe After Effects (Adobe Systems Inc., 2018), and by replacing them with a uniform gray colored background (RGB color code $[145,145,145])$. This led to a total of 60 two-second videos with a grey background showing ten individuals (five males and five females) per facial expression.

\subsection{Procedure}

Participants were brought to a quiet room, in which they were given written and verbal instructions about the experimental procedure. After filling in the informed consent form, electrodes were attached to the participants' faces for the facial electromyography recordings (see Measurements sections). During the tasks, participants were seated in $31.4 \mathrm{~cm}$ distance of a Philips screen with a resolution of 1920 x 1080 pixels, on which the stimuli (720 x 480 pixels) were presented using EPrime 3.0 software (Psychology Software Tools, Pittsburg, PA). The grey background colour of all screens was set to the background colour of the stimuli (RGB color code $[145,145,145])$. The same 60 emotional facial expression videos were presented in two consecutive tasks, a passive viewing task during which the participants' facial muscle activity was recorded and a facial emotion recognition task. In the passive viewing task, participants were instructed to only view the stimuli without performing any action. Each trial started with the presentation of a black fixation cross against a grey background for one second, and was followed by one of the 60 video stimuli for two seconds. The end 
of a trial was marked by a grey inter-trial interval (ITI) screen which appeared with a jittered duration of either 5750, 6000, or 6250ms. Participants viewed each of the 60 videos twice, in two separate blocks, resulting in 120 trials in total. Between the blocks, participant could take a self-paced break. The passive viewing task lasted around 20 minutes in total. After the passive viewing task which lasted approximately 20 minutes, the electrodes were detached from the participant's faces and the experiment continued with the facial emotion recognition task. In this second task, the participants viewed all 60 video stimuli once again (thus three times in total) but were now instructed to answer questions about them. Similar to the passive viewing task, each trial started with the fixation cross screen for one second and one of the 60 video stimuli (2s) was presented afterwards. Once the video disappeared, participants were asked to judge the displayed expression. More specifically, on the first question screen, they were asked to rate the expression according to its representativeness of the six expression categories which could be displayed: anger, fear, happiness, neutral, sadness, and surprise ('To what degree does the expression relate to the emotions below?'). Each expression category was accompanied by horizontal sliders ranging from 'not at all' to 'very much' and participants had to move the slider to indicate their judgment. The values of the sliders ranged from 0 to 100 in steps of $10(0$, 10, 20, etc.) which were not visible to the participants. The next screen contained three questions: (1) 'How intense was the expression displayed in the video?' to measure perceived emotional intensity; (2) 'Are you confident about your decision?' to measure confidence; and (3) 'Did you find the trial simple?' to measure simplicity (Zwick \& Wolkenstein, 2017). Again, all questions were accompanied by a slider ranging from 'not at all' to 'very much' with underlying values ranging from 0 to 100 in steps of 10. Thus, higher scores indicated higher ratings on perceived intensity, confidence, and simplicity, respectively. After the second question screen, a grey inter-trial interval (ITI) screen appeared for three seconds. In total, the participants completed 60 trials, rating all stimuli from the first task, plus three additional practice trials showing a different individual to familiarize them with the task. After 30 trials, participants could take a self-paced break and the entire facial emotion recognition task lasted approximately 25 to 30 minutes. A visualization of one facial emotion recognition trial can be found in Fig. 2. 
Importantly, only the ratings on the association of the displayed expression with the expression categories (first question screen) and the confidence ratings on the second screen were relevant to answer our hypotheses. As we did not formulate specific hypotheses about alterations in perceived emotional intensity ratings related to social anxiety or autistic traits, explorative analyses on this rating variable can be found in the Supplemental Materials. Further, the simplicity ratings provided insights in how difficult emotion recognition with this novel stimulus set was perceived. Overall, happy expressions received the highest simplicity ratings $(M=69.39, S D=27.15)$, followed by surprised expressions, $(M=62.51, S D=25.35)$, fearful expressions $(M=50.93, S D=26.19)$, angry expressions $(M=45.05, S D=25.66)$, neutral expressions $(M=36.42, S D=33.74)$, and sad expressions $(\mathrm{M}=36.19, \mathrm{SD}=25.56)$. Lastly, participants filled in the Liebowitz Social Anxiety Scale (LSAS; Liebowitz, 1987) and the Autism Spectrum Quotient (AQ; Baron-Cohen et al., 2001; see Measurements sections). Upon completion of the experiment, participants were given a written and verbal debriefing about the goal of the study and were reimbursed. In total, the experiment lasted around 55 minutes, including instructions and the attachment of the facial electromyography electrodes.

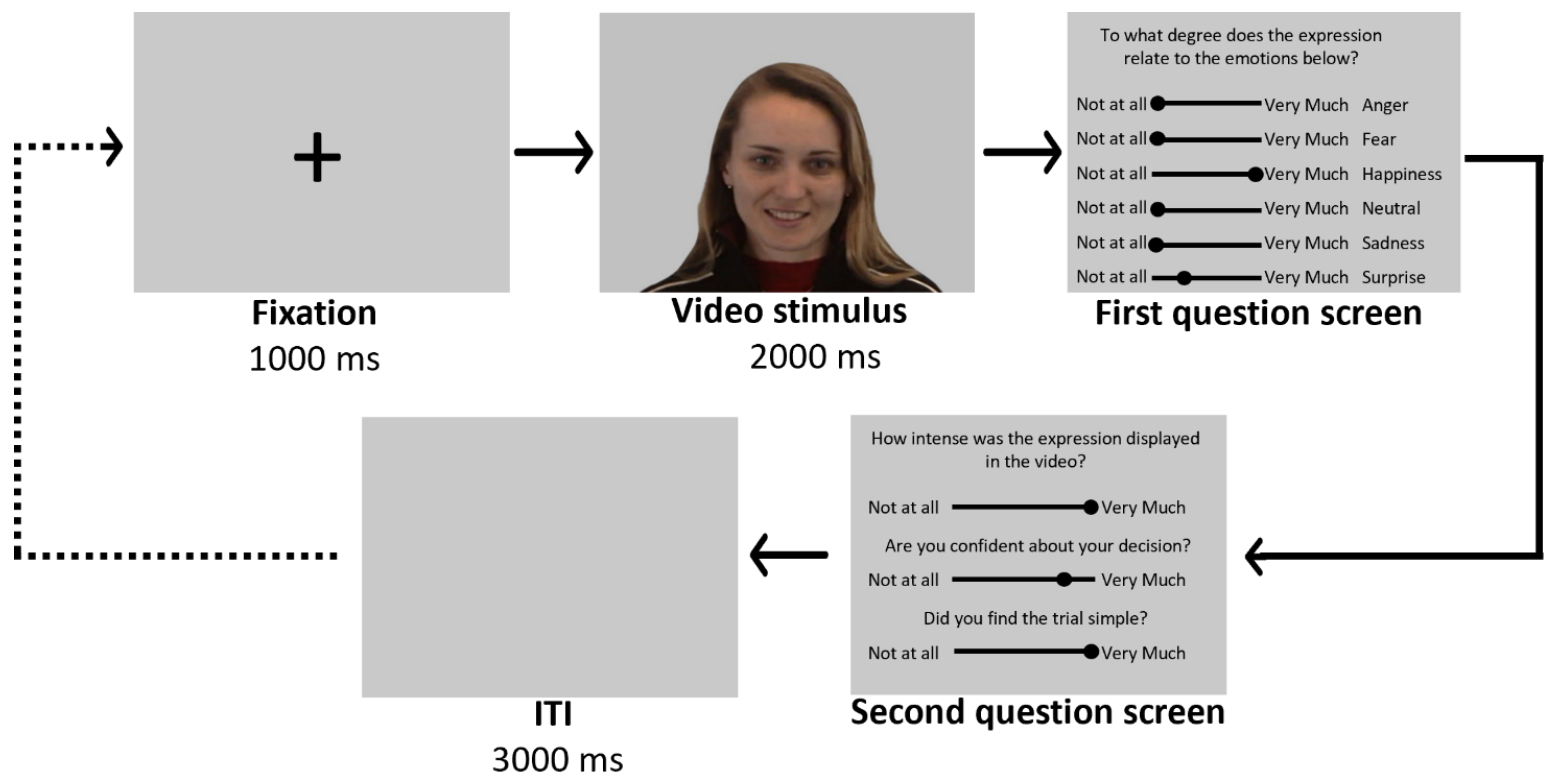

Fig. 2. Illustration of a Facial Emotion Recognition task trial. Each trial started with a fixation screen and ended with an intertrial interval (ITI) screen. The dotted line indicates that this sequence was repeated until all 60 videos were presented once. 


\subsubsection{Measurements}

\subsubsection{Facial electromyography (fEMG)}

Facial electromyography (fEMG) was measured on the left side of the face of all our participants, following the guidelines of Fridlund and Cacioppo (1986). To specify, two reusable 4 $\mathrm{mm} \mathrm{Ag/AgCl}$ surface electrodes were placed over the Corrugator Supercilii region and two over the Zygomaticus Major region. Additionally, a ground electrode was added on the top of the forehead. The signal was transmitted and amplified using a Biopac MP150 system combined with a BioNomadix 2 channel wireless EMG amplifier. Data recordings were made in AcqKnowledge 4.3 (Biopac Systems Inc.) using a sampling rate of $1000 \mathrm{~Hz}$. Event markers as defined in the E-Prime tasks (Psychology Software Tools, Pittsburg, PA) were sent via a parallel port and saved within an event marker channel. For data preprocessing, the EMG recordings were loaded into the PhysioDataToolbox in which they were rectified and filtered with a $28 \mathrm{~Hz}$ high cut-off filter, a $500 \mathrm{~Hz}$ low cut-off filter, and a $50 \mathrm{~Hz}$ notch filter. For each trial, separate epochs were defined for the fixation period (1s), the first $500 \mathrm{~ms}$ of stimulus presentation in which a neutral expression was shown (later defined as baseline), the subsequent $1500 \mathrm{~ms}$ in which the emotional expression was shown (later defined as response), and the first $1500 \mathrm{~ms}$ of the blank screen after stimulus presentation. Within these epochs, the EMG signal was downsampled by calculating the average signal within consecutive $100 \mathrm{~ms}$ time bins. The combined data from all subjects was then exported into MATLAB for further preprocessing. First, an automated artifact detection, which was inspired by Dignath et al. (2019), was conducted. More specifically, for each subject and each muscle region, we checked the distribution of the EMG signal for extreme values (+/- 3.5 SDs) in the time bins regarding the absolute value of each time bin and the relative differences between subsequent bins. This was performed in relation to (1) the entire time interval of interest per trial ( 5 baseline time bins and 15 response time bins) and (2) the distribution of baseline time bins in the same position across trials. If more than $50 \%$ of all time bins (20) or more than $50 \%$ of the baseline time bins (5) belonging to one trial had extreme values, this trial was entirely excluded from the analysis. Otherwise, the respective time bins were replaced with missing values. Across all subjects, 17 trials $(0.002 \%)$ were excluded from the corrugator analysis and 
150 trials $(0.02 \%)$ from the zygomaticus analysis. After excluding the marked time bins, a baseline correction was performed by subtracting the mean EMG activity of all baseline time bins belonging to one trial from the respective response time bins. The baseline-corrected EMG data was then z-scored for each participant and each muscle region. Further, the data was summarized on a trial level by averaging the last second of each trial's response window (last 10 time bins) for each participant and each muscle region as well as by averaging across the two presentations of each of the 60 stimuli to end up with the same amount of observations as for the rating data (trial-averaged data; used as predictor in generalized linear mixed models). Lastly, the data was also summarized on a participant level by creating the average of the same time window across trials for each participant, each muscle region and each emotion category (category-averaged, used as outcome variable in linear models).

\subsubsection{Questionnaires}

Social anxiety measure. We used the Liebowitz Social Anxiety Scale (LSAS; Liebowitz, 1987) to measure self-reported social anxiety traits in our non-clinical sample. The LSAS is designed to assess fear and avoidance levels of individuals with social phobia in a range of social interaction and performance scenarios. The questionnaire contains 24 items in total. Respondents score the items for fear and avoidance separately on a 3-point Likert scale, 0 (= None), 1 (= Mild), 2 (= Moderate), 3 (= Severe). The scores are all added up to a total sum of all subscales, with higher scores indicating a higher severity of social anxiety symptoms. LSAS scores in our sample ranged from $7-73(M=$ $38.53, S D=17.53)$, with 30 participants $(52.63 \%)$ exceeding the cut-off of 30 , indicating a probability of social anxiety (Mennin et al., 2002; Rytwinski et al., 2009). With a skewness of 0.21 and a kurtosis of 2.08, the distribution of the LSAS scores showed to be slightly platykurtic, yet close to normal (see Fig. 3A).

Autism Measure. The Autism-Spectrum Quotient (AQ; Baron-Cohen et al., 2001), as used in our study, is a self-report questionnaire which was created to measure traits associated with the autism spectrum. The AQ consists of 50 items in total and can be divided into five subscales (10 items each) assessing different domains: social skill, attention switching, attention to detail, communication, and imagination. Respondents indicate how strongly each item applies to them based on a 4-point Likert 
scale ranging from 1 (= definitely agree), 2 (= slightly agree), 3 (= slightly disagree), and 4 (definitely disagree), and some items are reversely scored to prevent response biases. All item scores are added up to a total sum score, with higher scores reflecting higher autistic trait levels. The range of AQ scores was between $2-39$ in our sample $(M=16.47, S D=7.34)$ and only 3 participants $(5.26 \%)$ had a higher AQ score than 32 which indicates autistic trait levels of clinical significance. The skewness and kurtosis of the AQ score distribution were 1.05 and 4.17 respectively, thus showing a positive skew (see Fig. 3B).
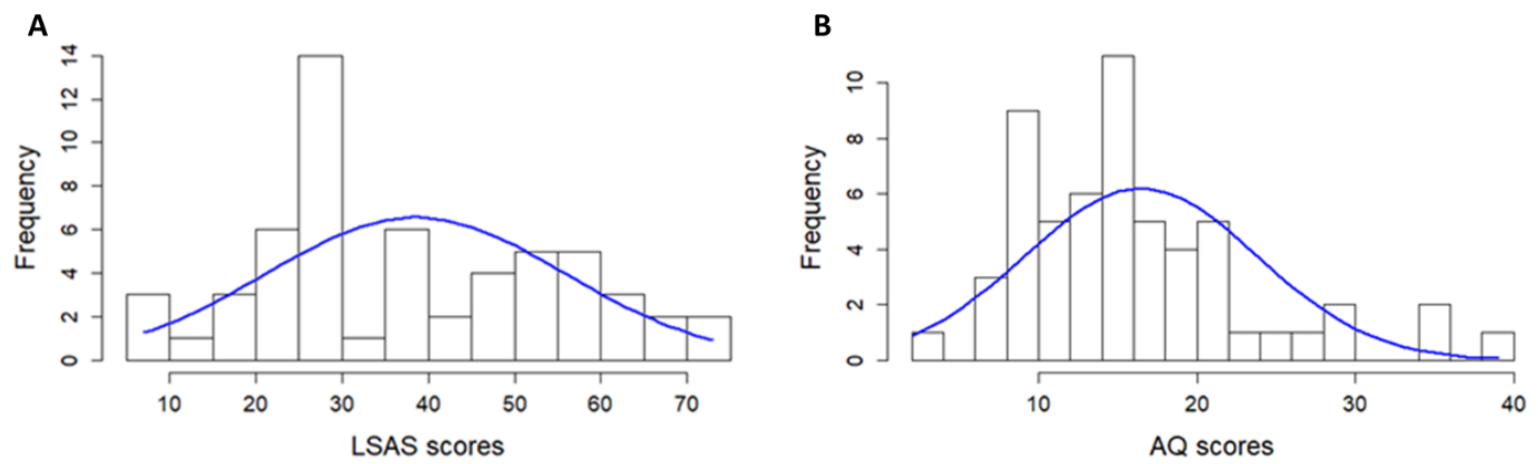

Fig. 3. Distribution of (A) social anxiety traits (LSAS scores) and (B) autistic traits (AQ scores) in our sample. The blue line indicates the estimated normal distribution, based on the mean scores, standard deviations, minimum and maximum. 


\subsection{Data analysis}

Spearman's rank correlation revealed that autistic traits and social anxiety traits, reflected by the scores on the two questionnaires, were not significantly associated with each other, $r_{s}=0.04, p=$ 0.784. Our sample showed both sufficient variance in and independence between the trait dimensions, allowing for separate analyses for the two trait dimensions .Emotion recognition accuracy was calculated by determining the expression category with the highest slider score and comparing it to the predefined category of the stimulus for each trial (see Zwick \& Wolkenstein, 2017). If there was a match between the presented and the perceived expression category, a trial was scored as correct (1) whereas it was scored as incorrect (0) in case of a mismatch. Trials in which two expression categories received the same slider scores were discounted from the analysis (missing values). To check the robustness of this approach, we re-ran all analyses on accuracy with a relative accuracy score which was calculated by subtracting the mean score of all other expression categories from score of the correct expression category (Keating et al., 2021). The results are reported in the Supplemental Materials. All analyses were performed in R 3.6.3 (R Core Team, 2020), using the lmerTest package (Kuznetkova et al., 2017) for fitting the (generalized) linear mixed models ([G]LMMs), the multcomp package (Hothorn et al., 2008) for general hypotheses testing, the sjPlot package (Lüdecke, 2021) for creating the tables and both the sjPlot package and ggplot2 (Wickham, 2016) for creating the plots.

\subsubsection{Behavioural analysis}

Accuracy and Confidence. In order to test whether social anxiety traits were associated with better emotion recognition performance for negative expressions and whether emotion recognition accuracy was generally reduced with higher autistic traits, we calculated two binomial GLMMs on accuracy with emotion category, the respective trait dimension and their interaction as predictors. Both participant ID and the identity of the stimulus were added as random effects (random intercept). Further, we fitted two LMMs on emotion recognition confidence to test whether higher social anxiety traits were indeed associated with an underconfidence in emotion recognition, and to explore potentially altered confidence judgments with higher autistic trait levels. The models had the same fixed and random effects structure as the accuracy models. Coefficients for the emotion categories 
(main effects and interactions) were calculated by contrasting the respective category against the overall effect. For the neutral category, coefficients were calculated and tested (z-tests) using general hypotheses testing.

Metacognitive sensitivity. To examine how well an individual's confidence ratings could distinguish between accurate and inaccurate trials in the emotion recognition task, we calculated the hit and false alarm rate pairs with increasing confidence levels (11, according to points on the Likert scale) for each subject and employed the area under the type 2 ROC curve (AUROC2) approach according to Fleming and Lau (2014). More specifically, each confidence level was taken as a criterion to distinguish between low and high confidence trials; starting with a criterion in which only zeroes were regarded as low confidence ratings and all higher values were regarded as high confidence ratings, up until a criterion in which all trials below the highest confidence rating (100) were regarded low confidence trials and only the highest rating was regarded as high confidence. The resulting probabilities for hits, $\mathrm{p}$ (high confidence|correct), and false alarms, $\mathrm{p}$ (high confidence|incorrect), were plotted against each other for each confidence level. The resulting area under this ROC 2 curve was taken as an index for the subject's metacognitive sensitivity, describing how well an individual's confidence ratings were scaled to actual emotion recognition accuracy. The link to each clinical trait was then tested with a correlational analysis.

Link between confidence and emotion recognition. For a description of inter-individual variation in the relationship between confidence and emotion recognition, we zoomed in on a trial level and tested whether the coupling between the two variables was altered depending on an individual's clinical trait levels as well as the presented expression. Therefore, the two GLMMs on emotion recognition accuracy were extended by adding confidence ratings and all 2-way interactions as well as the 3-way interaction with emotion category and the respective clinical trait dimension to the models. Coefficients for the emotion categories (main effects and interactions) were once again calculated by contrasting the respective category against the overall effect. For the neutral category, coefficients were calculated and tested (z-tests) using general hypotheses testing. 


\subsubsection{Facial EMG analysis}

Facial muscle activity (Mimicry). In order to test whether social anxiety traits are associated to an enhanced mimicry of specifically angry (negative) expressions, we fitted a linear model on the category-averaged corrugator activity with emotion category, social anxiety traits and their interaction as predictors. We also aimed to explore zygomaticus activity for mimicry of happy expressions and, therefore, used the same independent variables to predict category-averaged zygomaticus activity. By replacing social anxiety traits with autistic traits in the other two linear models on categoryaveraged corrugator and zygomaticus activity, we then tested whether typical mimicry patterns are indeed reduced with higher autistic traits (i.e., less corrugator activity for negative expressions (specifically anger), less zygomaticus activity for happy expressions + less decrease in corrugator activity for happy expressions). Coefficients for the emotion categories (main effects and interactions) were calculated by contrasting the respective category against the neutral reference category. Since the residual plots of the two model fits on zygomaticus activity showed violation of the normality assumption as well as heterogeneous error estimates, we calculated non-parametric estimates of the predictor effects and confidence intervals, using 1000 bootstrap iterations, for these two models.

\section{Link between facial muscle activity (mimicry) and emotion recognition. We investigated the} relationship between intraindividual differences in facial muscle activity and emotion recognition accuracy in the context of varying clinical trait levels by running separate analyses for the emotion categories anger, happiness, sadness and fear. Binomial GLMMs were fitted on emotion recognition accuracy with the trial-averaged EMG activity over the two muscle regions (corrugator and zygomaticus) as distinct predictors, as well as one of the clinical trait scores and both two-way interactions ( 8 models in total). Similar to the behavioural accuracy models, random intercepts were added for the subject as well as the stimulus identity. 


\section{Results}

\subsection{Behavioural results}

\subsubsection{Accuracy in ER}

Descriptive statistics. Emotion recognition performance differed by emotion category with highest values obtained in the recognition of expressions of happiness $(M=0.97, S D=0.17)$, then surprise $(M=0.91, S D=0.29)$, neutral $(M=0.90, S D=0.31)$, anger $(M=0.76, S D=0.42)$, sadness $(M=0.51, S D=0.50)$ and lastly fear $(M=0.25, S D=0.43)$. Importantly, recognition rates only include expressions for which a distinct classification was made (i.e. not expressions which were equally rated on two emotion categories).

Social anxiety traits. In the first binomial GLMM on emotion recognition accuracy including emotion category, social anxiety traits and their interaction as predictors, emotion category significantly predicted accuracy, $\chi 2(5)=702.730, p<.001$. Emotion recognition performance for happy, surprised and neutral expressions was significantly better than average, happy: $\mathrm{OR}=10.836, \mathrm{z}$ $=10.790, \mathrm{p}<.001$, surprise: $\mathrm{OR}=3.031, \mathrm{z}=7.652, \mathrm{p}<.001$, neutral: $\mathrm{OR}=2.336, \mathrm{z}=6.415, \mathrm{p}<.001$. In contrast, sad and fearful expressions were significantly worse recognized than average, $\mathrm{OR}=0.232$, $\mathrm{z}=-14.342, \mathrm{p}<001$, and $\mathrm{OR}=0.066, \mathrm{z}=-23.441, \mathrm{p}<.001$ respectively. Thus, we did not find that the association between emotion categories and accuracy varies depending on social anxiety trait levels.. An overview of the model fit can be found in the Supplemental Material (see Table S1).

Autistic traits. Similar to the first model, emotion category was a significant predictor in the second binomial GLMM on emotion recognition accuracy, $\chi 2(5)=679.873, p<.001$, as were autistic traits, $\chi 2(1)=9.103, p=.003$, with higher trait levels predicting a worse performance, $\mathrm{OR}=0.766$. The significant interaction between the two variables, $\chi 2(5)=17.641, p=.003$, revealed that the overall negative association between autistic traits and emotion recognition accuracy was most pronounced for fearful expressions, $\mathrm{OR}=0.683, \mathrm{z}=-2.928, p=.003$. In contrast, recognition of sad expressions was less negatively affected by autistic traits, $\mathrm{OR}=1.272, \mathrm{z}=2.459, p=.014$, compared to the overall performance (for more information, see Fig. 4b and Table S2 in the Supplemental 
Material). Thus, the expected overall negative association between autistic traits and emotion recognition performance seems specifically pronounced for fearful facial expressions.
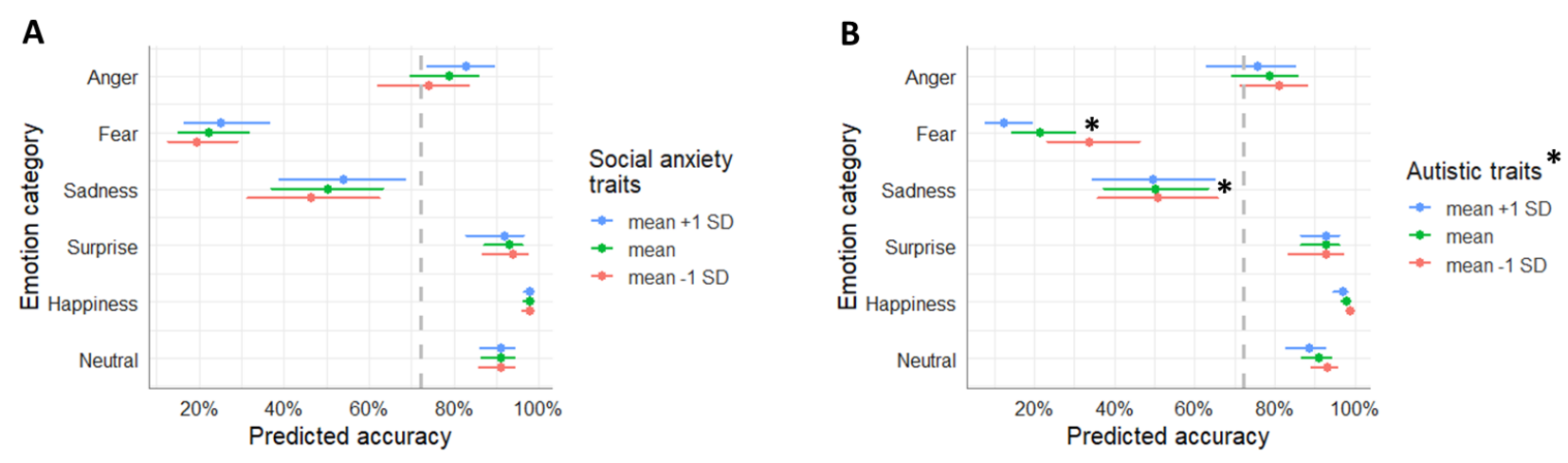

Fig. 4. Predicted emotion recognition accuracies depending on (A) social anxiety trait levels and (B) autistic trait levels by emotion category (anger, fear, sadness, surprise happiness, neutral). For illustrative purposes, predicted accuracies for mean values as well as mean values $+/-1 \mathrm{SD}$ of the continuous variables social anxiety traits and autistic traits are depicted. Whiskers represent confidence intervals and significant effects are marked with an asterisk.

\subsubsection{Confidence in ER}

Descriptive statistics. Highest values for confidence judgments in emotion recognition depending on the emotion category were given to stimuli belonging to the category happiness $(M=$ 83.23, $S D=23.49)$, then neutral $(M=70.78, S D=28.95)$, then surprise $(M=67.60, S D=26.10)$, then anger $(M=57.81, S D=30.88)$, then fear $(M=56.59, S D=28.02)$, and lastly sadness $(M=54.58, S D$ $=28.81)$.

Social anxiety traits. The first LMM on confidence in emotion recognition, with emotion category, social anxiety traits and their interaction as predictors, revealed a negative association between social anxiety traits and confidence, $F(1,3344)=5.897, p=0.018, \beta=-0.138$. Thus, independent of the displayed expression, confidence judgments were significantly lower with higher social anxiety trait levels. Further, confidence judgments also varied by emotion category, $F(5,3344)$ $=2239.774, p<.001$, but this variation was independent of social anxiety traits (i.e. no interaction). 
Overall, participants were most confident in judging displays of happiness, $\beta=0.614, t(3344)=$ $19.696, p<.001$, and significantly more confident in judging neutral and surprise expressions than overall, $\beta=0.193, \mathrm{z}=6.186, \mathrm{p}<.001$ and $\beta=0.084, t(3344)=2.699, p=.007$, respectively. For the other emotional expressions, confidence was significantly reduced, angry: $\beta=-0.247, t(3344)=-$ 7.933, $p<.001$, fearful: $\beta=-0.287, t(6761)=-9.210, p<.001$, sad: $\beta=-0.356, t(3344)=-11.439, p<$ .001. (see Fig. 5a and Table S3 in the Supplemental Material).

Autistic traits. In the LMM which included autistic traits next to emotion category and their interaction to predict confidence in emotion recognition, emotion category was again a significant predictor, $F(5,3344)=120.420, p<.001$, as well as the interaction between emotion category and autistic traits, $F(5,3344)=10.695, p<.001$. While for neutral and happy facial expressions, confidence was significantly reduced with higher autistic traits, $\beta=-0.156, \mathrm{z}=-5.047, \mathrm{p}<.001$, and $\beta$ $=-0.108, \mathrm{t}(3344)=-3.468, \mathrm{p}=.001$ respectively, this effect reversed for displays of fear and sadness. In those two categories, autistic traits were associated with higher confidence ratings, fear: $\beta=0.107$, $t(3344)=3.435, p=.001$; sadness: $\beta=0.116, t(3344)=3.732, p<.001($ see Fig. $5 \mathrm{~b}$ and Table $\mathrm{S} 4$ in the Supplemental Material).
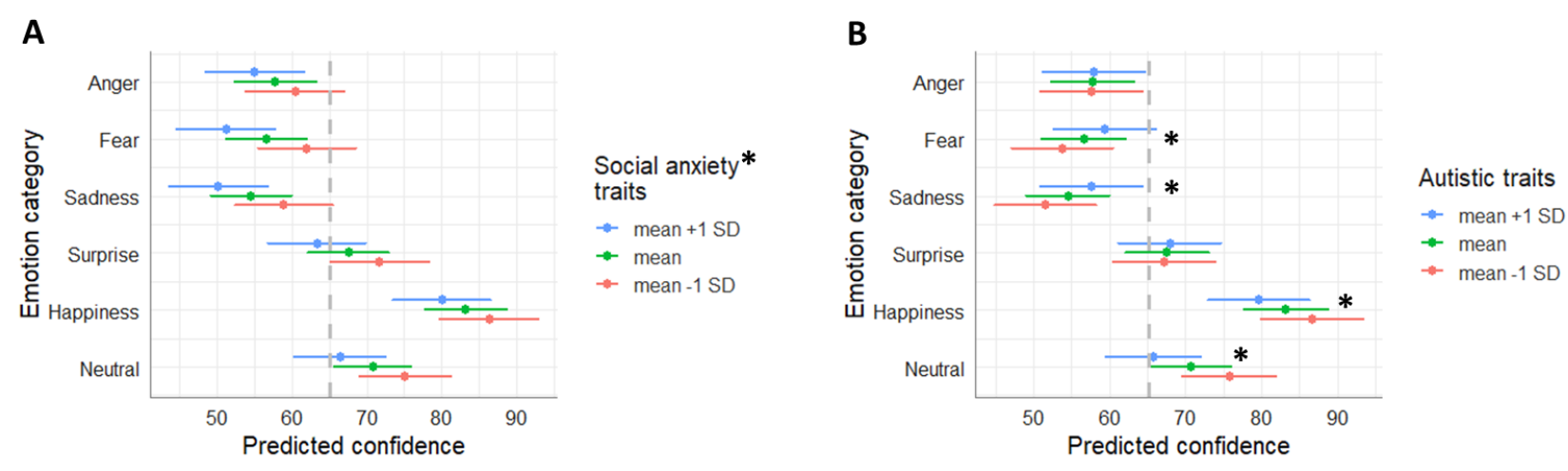

Fig. 5. Predicted confidence in emotion recognition depending on (A) social anxiety trait levels and (B) autistic trait levels by emotion category (anger, fear, sadness, surprise happiness, neutral). For illustrative purposes, predicted accuracies for mean values as well as mean values $+/-1 \mathrm{SD}$ of the continuous variables social anxiety traits and autistic traits are depicted. Whiskers represent confidence intervals and significant effects are marked with an asterisk. 


\subsubsection{Metacognitive sensitivity}

According to Mahalanobis distances, two participants had to be excluded from the correlation between social anxiety traits and AUROC2 and four participants had to be excluded from the analysis between autistic traits and AUROC2. After excluding these bivariate outliers, all distributions did not majorly deviate from normality. The two correlational analyses between the clinical trait dimension and metacognitive sensitivity (AUROC2) revealed that autistic traits, but not social anxiety traits, were significantly related to metacognitive sensitivity (see Fig. 6). As expected, metacognitive sensitivity was reduced with higher autistic traits, $r=-.482, t(51)=-3.939, p<.001$ and $r_{s}=-.522, \mathrm{p}<.001$. For social anxiety, $r=-.208, t(53)=-1.549, p=.127$ and $r_{s}=-.245, p=.072$.
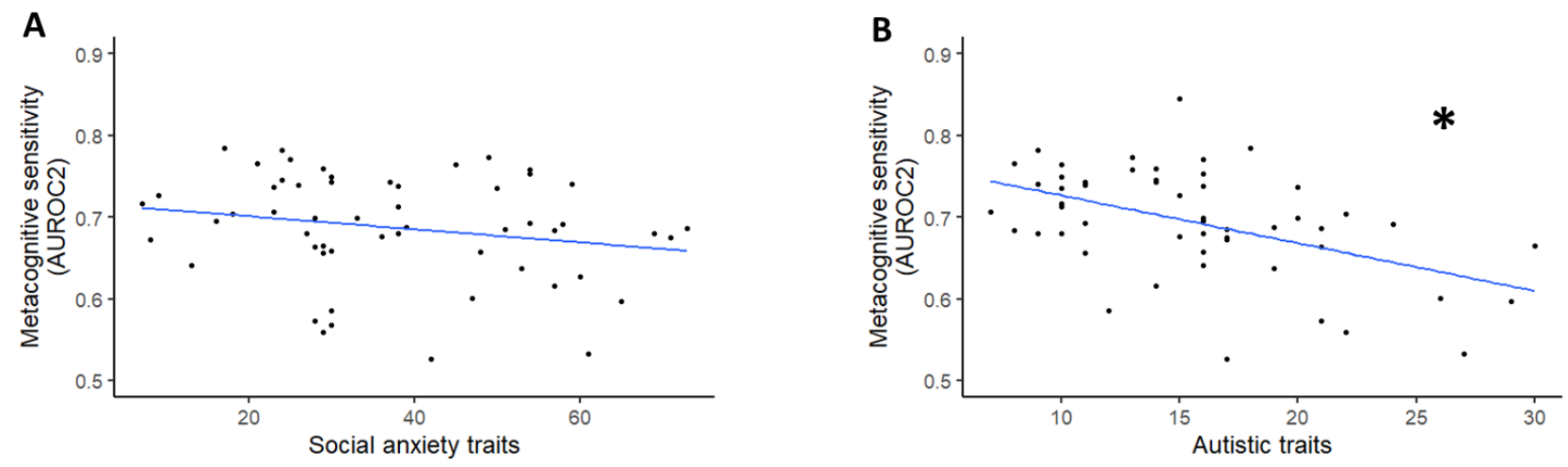

Fig. 6. Relationship between (A) social anxiety straits or (B) autistic traits and metacognitive sensitivity, i.e. area under the type 2 ROC curve (AUROC2). The blue line indicates the estimated linear relationship, with significant relationships being marked by an asterisk.

\subsubsection{Link between confidence and emotion recognition}

Social anxiety traits. When exploring the link between confidence and accuracy in emotion recognition in relation to the clinical trait dimensions in more detail, on a trial level and by emotion category, social anxiety traits similarly did not seem to have a substantial impact on the link. More specifically, only confidence, emotion category and the interaction between the two were significant predictors of emotion recognition accuracy, confidence: $\chi^{2}(1)=81.919, \mathrm{p}<.001, O R=1.932$, category: $\chi^{2}(5)=605.610, \mathrm{p}<.001$, confidence* category: $\chi 2(5)=23.861, \mathrm{p}<.001$. The overall 
positive relationship between confidence and accuracy was more clearly pronounced for angry and happy, $O R=1.435, z=2.793, p=.005$ and $O R=1.616, z=2.113, p=.035$ respectively, and less clearly pronounced for fearful and surprised facial expressions, $O R=0.687, z=-3.120, p=.002$ and $O R=0.685, z=-2.275, p=.023$ respectively (see Fig. 7). An overview of all model parameters can be found in Table S5 in the Supplemental Material.

Autistic traits. Similarly, when including autistic traits in the GLMM, no influence of trait levels on the link between confidence and emotion recognition became apparent. In this model, emotion recognition accuracy was significantly predicted by confidence, emotion category, and autistic traits, confidence: $\chi^{2}(1)=79.863, \mathrm{p}<.001, O R=1.917$, category: $\chi^{2}(5)=597.242, \mathrm{p}<.001$, autistic traits: $\chi 2(1)=9.139, \mathrm{p}=.003, O R=0.747$. Further, the interaction between emotion category and autistic traits, as well as the interaction between emotion category and confidence, $\chi 2(5)=16.901, p=.005$, and $\chi^{2}(5)=23.003, \mathrm{p}<.001$ respectively (see Table S6 in the Supplemental Material). In line with the model only including autistic traits and emotion category in the prediction of accuracy, the generally reduced emotion recognition accuracy was more pronounced for fearful expressions, $O R=$ $0.636, z=-3.138, p=.002$, and less pronounced for sad expressions, $O R=1.284, z=2.311, p=.021$. Moreover, as in the above-described model, accurate recognition of angry and happy expressions was more strongly (i.e. positively) linked to confidence ratings, $O R=1.391, z=2.619, p=.009$ and $O R=$ $1.640, z=2.192, p=.028$ respectively, whereas the link was weaker for fearful and surprised expressions, $O R=0.680, z=-3.119, p=.002$ and $O R=0.695, z=-2.251, p=.024$ respectively. 


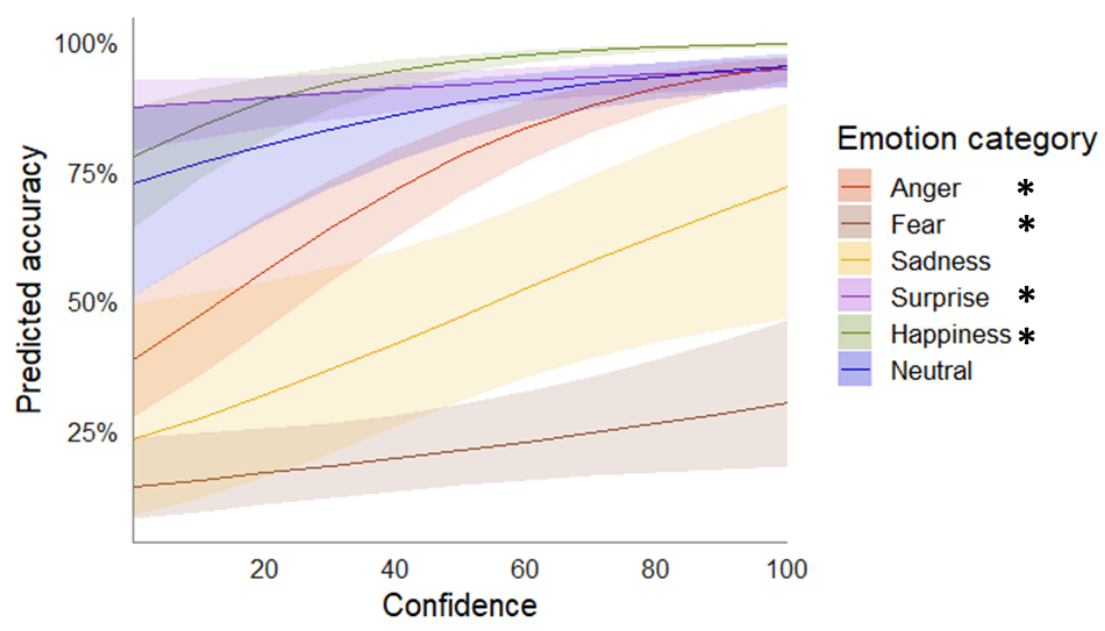

Fig. 7. Predicted accuracies depending on confidence in emotion recognition by emotion category (anger, fear, sadness, surprise happiness, neutral), taken from the model including social anxiety traits. Shaded areas represent confidence intervals and significant effects are marked with an asterisk in the legend.

3.2. Facial Electromyography (fEMG) results

\subsubsection{Facial mimicry}

In all models with category-averaged and z-scored EMG activity in the last second of the response window as an outcome and emotion category, clinical traits (social anxiety or autism) and their interaction as predictors, neither main effects of clinical traits nor interaction effects with emotion category were found. Consequently, we did not find evidence for alterations in emotion-specific facial responses, and also general facial expressiveness associated to social anxiety traits or autistic traits. We did, however, find significant effects of emotion category on EMG activity which, based on the social anxiety models, will be reported below (comparable results for the autistic trait model can be found in Tables S8 and S10 in the Supplemental Material).

Corrugator. As expected, activation of the corrugator depended on the displayed emotion category, $F(5,330)=70.025, p<.001$. In line with previous research, when compared to neutral 
expressions, corrugator activity to angry expressions was found to be enhanced, $\beta=0.117, \mathrm{t}(330)=$ $2.874, \mathrm{p}=0.004$, whereas activity to happy expressions was found to be diminished, $\beta=-0.547, t(330)$ $=-13.432, p<.001$. Surprisingly, displays of surprise were also related to a reduced corrugator activity, $\beta=-0.205, t(330)=-5.032, p<.001$ (see Fig. 8 and Table S7 in the Supplemental Material).

Zygomaticus. Given the non-normal distribution of the model residuals, exclusively coefficients estimated by post-hoc bootstrapping analyses were considered. Following our expectations, zygomaticus activity was strongly enhanced for happy expressions compared to neutral expressions, B $=0.542,95 \% \mathrm{CI}[0.394,0.707], \mathrm{p}=.001$, indicating a mimicry of smiles. Additionally, the zygomaticus was more strongly activated for surprised versus neutral facial expressions, $B=0.073$, 95\% CI $[0.012,0.128], \mathrm{p}=.017$ (see Fig. 8 and Table S9 in the Supplemental Material).

A

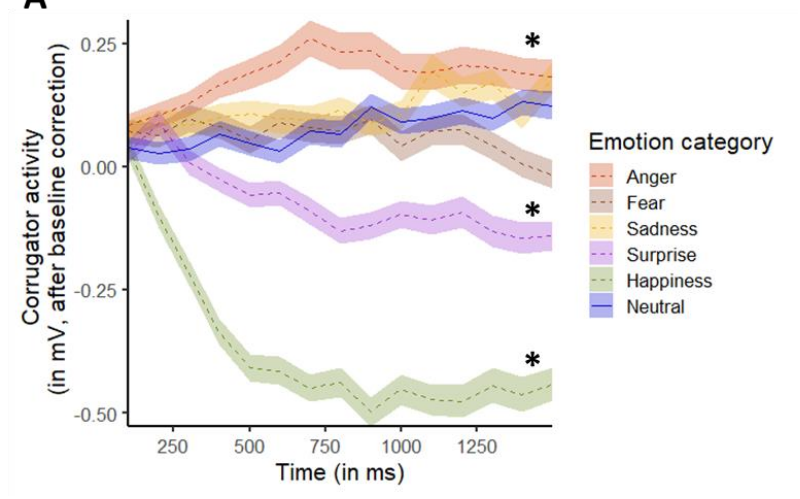

B

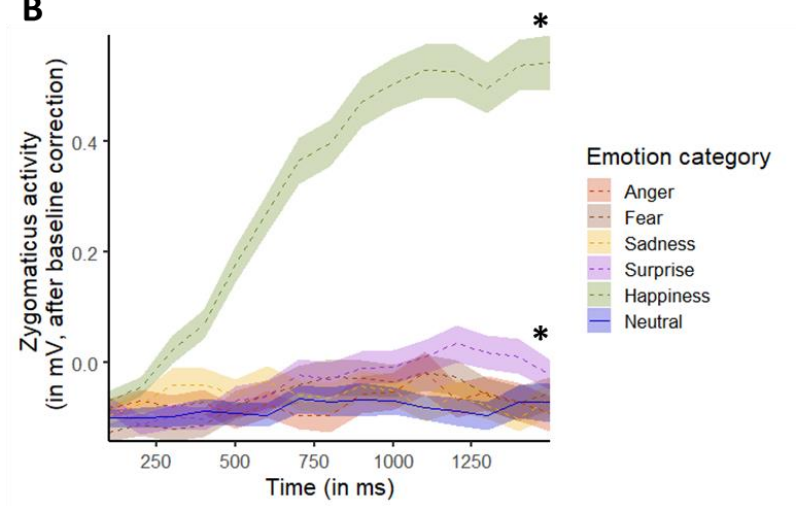

Fig. 8. Time course of the filtered, baseline-corrected and z-scored facial electromyography (EMG) signal over (A) the Corrugator Supercilii region and (B) the Zygomaticus Major region related to the passive viewing of the naturalistic facial expressions by emotion category. The mean of the first $500 \mathrm{~ms}$ of each $2 \mathrm{~s}$ video (neutral expression) was taken as a baseline and only the mean EMG response in the last second of stimulus presentation (full-blown expression, 500ms $-1500 \mathrm{~ms}$ in plot) was considered in the facial EMG analysis. The coloured shaded areas around the values indicate standard errors of the means and asterisks mark statistical significance. 


\subsubsection{Facial mimicry in emotion recognition}

Social anxiety traits. For each emotion category in which mimicry was expected (angry, happy, fearful and sad), separate binomial GLMMs were fitted with emotion recognition accuracy as outcome and corrugator activity, zygomaticus activity, social anxiety traits, and two-way interactions between each muscle activity and social anxiety traits as predictors. None of these predictors was significantly associated with emotion recognition accuracy in the models on angry and fearful displays (see Tables S11 and S13 in the Supplemental Material). The model on sad expressions revealed that accuracy was higher when the corrugator muscle was more strongly activated, $\chi 2(1)=4.462, \mathrm{p}=0.035, \mathrm{OR}=1.574$ (Table S14 in the Supplemental Material). Mimicry of sad facial expressions, as indicated by increased corrugator activity, might therefore indeed promote the recognition of sadness. Further, both zygomaticus activity as well as its interaction with social anxiety traits were significant predictors in the model on happy expressions, $\chi^{2}(1)=4.355, \mathrm{p}=.037$, OR $=6.179$, and $\chi^{2}(1)=-2.086, \mathrm{p}<.001$, $\mathrm{OR}=0.210$ respectively (Table S12 in the Supplemental Material). Hence, while the significant effect of zygomaticus activity hints towards a facilitating role of mimicry of smiles in emotion recognition, this link seems to be weakened with higher social anxiety traits. When examining the predicted value plot (see Fig. 9A), this effect, however, seems to be mainly driven by stronger variation in accuracies (i.e. also inaccurate responses) in individuals with lower social anxiety traits when the zygomaticus was not strongly activated. Otherwise, recognition of happy expressions was at ceiling and not much variation in relation to social anxiety trait levels could be observed.

Autistic traits. The separate binomial GLMMs with emotion recognition accuracy as outcome and corrugator activity, zygomaticus activity, autistic traits, and two-way interactions between each muscle activity and autistic traits as predictors, yielded slightly different results. While recognition of angry facial expressions was again not significantly related to any predictor, recognition of fearful expressions was lower with higher autistic traits, $\chi 2(1)=9.493, \mathrm{p}=0.002, \mathrm{OR}=0.460$ (see Tables S15 and S17 in the Supplemental Material). In addition, the model on the recognition of sad expressions showed significant effects of corrugator activity as well as a significant interaction between corrugator activity and autistic traits. More specifically, in line with the social anxiety model, 
sad facial expressions were better recognized with higher corrugator activity, $\chi 2(1)=4.569, p=0.033$, $\mathrm{OR}=1.596$. This relationship was, however, weaker for higher autistic traits, interaction: $\chi^{2}(1)=$ $4.178, \mathrm{p}=0.041, \mathrm{OR}=0.666$ (see Fig. 9B and Table S28). Further, significant effects of zygomaticus activity and of corrugator activity as well as a significant interaction between zygomaticus activity and autistic traits were found in the model on happy expressions, zygomaticus: $\chi 2(1)=5.609, p=0.018$, $\mathrm{OR}=13.192$, corrugator: $\chi 2(1)=4.887, \mathrm{p}=0.027, \mathrm{OR}=0.073$, autistic traits*zygomaticus: $\chi 2(1)=$ $5.853, \mathrm{p}=0.016, \mathrm{OR}=0.140$. In addition to the positive association between zygomaticus activity and recognition of happy expressions as described above, a negative relationship between corrugator activity and accuracy was observed. Thus, relaxation of the corrugator might additionally facilitate the recognition of happy expressions. Similar to the model including social anxiety traits, autistic traits had a negative effect on the positive association between zygomaticus activity and accuracy in the recognition of happy expressions (see Fig. 10C and Table S16 in the Supplemental Material). This interaction again seemed to be driven by cases of low zygomaticus activity associated with inaccurate responses, but this time linked to low autistic trait levels. Taken together, these results suggest that a relaxation of the corrugator muscle might support the recognition of happy facial expressions. Additionally, there might be facilitating effects of an activation of the corrugator muscle in the recognition of sad facial expression as well as the zygomaticus muscle in the recognition of happy facial expressions, which however, seem to be weaker with higher autistic traits levels. 

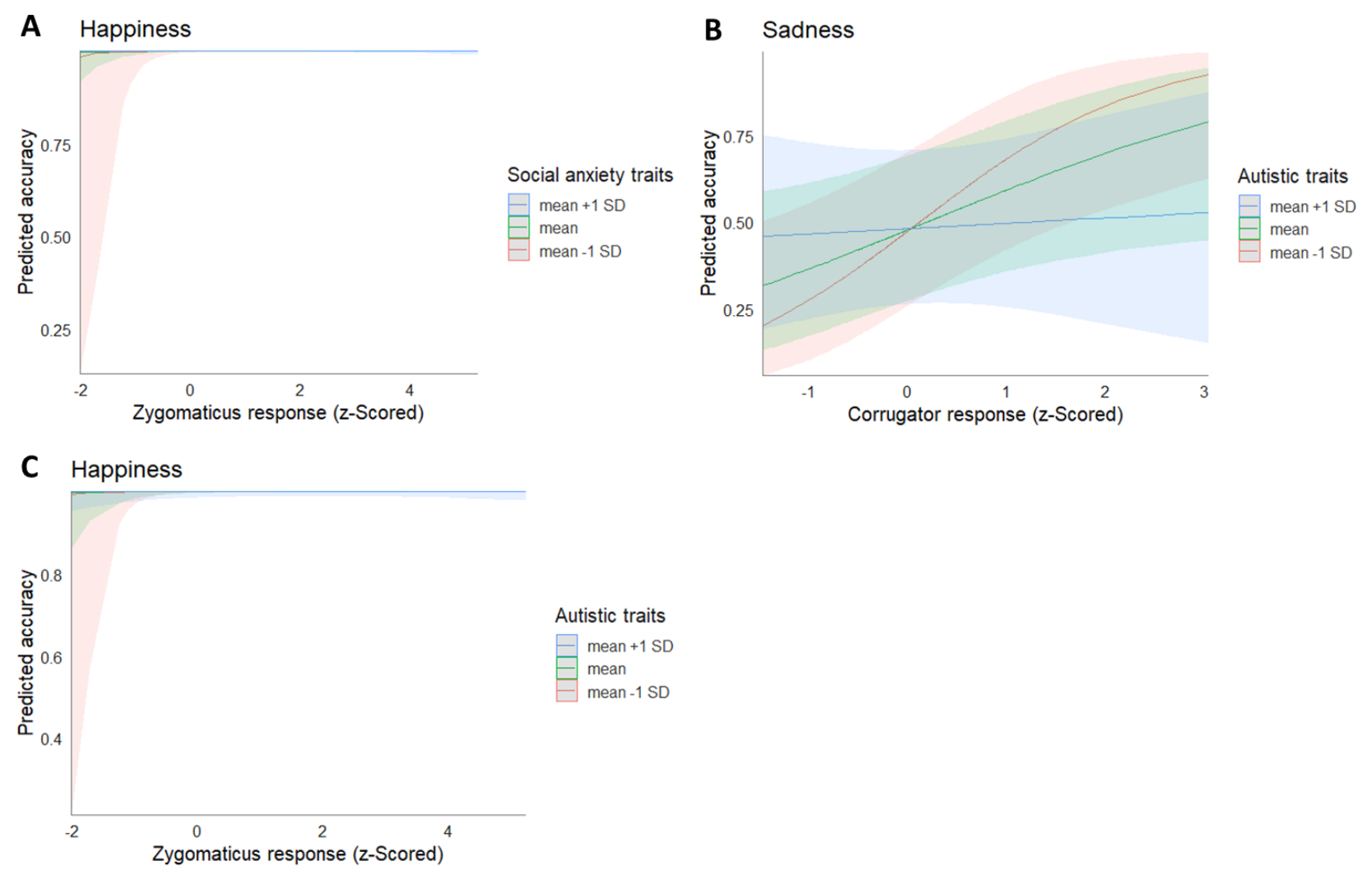

Fig. 9. (A) The relationship between predicted accuracy in recognizing happy expressions and the corresponding filtered, baseline-corrected and z-scored zygomaticus activity depending on social anxiety trait levels. (B) The relationship between predicted accuracy in recognizing sad expressions and the corresponding filtered, baseline-corrected and z-scored Corrugator activity depending on autistic trait levels. (C) The relationship between predicted accuracy in recognizing happy expressions and the corresponding filtered, baseline-corrected and z-scored zygomaticus activity depending on autistic trait levels. For illustrative purposes, predicted accuracies for mean values as well as mean values +/- 1 SD of the continuous variables social anxiety traits and autistic traits are depicted. Shaded areas represent confidence intervals. 


\section{Discussion}

In our study, we provided evidence that autistic traits and social anxiety traits are distinctly related to bottom-up and top-down components in emotion perception. Specifically, while individuals with higher autistic traits showed deficits in overall emotion recognition, and in particular for fearful expressions, individuals with higher social anxiety traits had significantly less confidence in their judgments regarding all emotion categories, despite an unaltered emotion recognition performance. Further, individuals with higher autistic traits seemed to be less able to calibrate their confidence judgments to their actual emotion recognition performance, i.e. displaying a poorer metacognitive sensitivity. While we did not observe alterations in emotion-specific facial muscle responses with regard to both clinical trait dimensions, we could replicate the most robust effects reported in the facial mimicry literature in our sample. Happy facial expressions were responded to with increased zygomaticus and decreased corrugator activity and angry facial expressions were responded to with increased corrugator activity. Moreover, we found indications that the mimicry of the corrugator might facilitate the recognition of sad expressions whereas the mimicry of expressions by the zygomaticus (and potentially relaxation of the corrugator) might support the recognition of happy expressions. Crucially, both links were less pronounced with higher autistic traits, while there was only weak evidence for a negative effect of social anxiety traits on the link between zygomaticus activity and accuracy in recognizing happy expressions.

\subsection{Modulations related to social anxiety traits}

Contradicting our expectations, we did not find a negativity bias with higher social anxiety traits reflected in our main analysis on the recognition accuracy of the displayed facial expressions. When using relative accuracy scores as an outcome (see Supplemental Material), however, a better recognition of angry facial expressions with higher social anxiety trait levels could be observed. Given that an improved recognition of negative expressions in SAD was also not consistently found in the literature (e.g. Bui et al., 2017), the effect seems unstable and additional factors might play a role. A previous study with high socially anxious individuals, for example, only found a recognition advantage for static but not dynamic facial expressions of anger (Torro-Alves et al., 2016). Further, a 
heightened sensitivity to negative expressions in social anxiety might only occur under brief exposure times or when actual interactions with the expresser could be expected (Staugaard, 2010). Importantly, social anxiety traits did have the expected impact on the confidence judgment with regard to emotion recognition in our study. For all expression categories, confidence was reduced with higher social anxiety traits. This reduction was also reflected in the perceived emotional intensity ratings which were generally lower with higher trait levels (see Supplemental Material). Individuals with higher social anxiety traits who had the feeling that they were less able to read an expression might have also classified them as less intense. The underconfidence in performance did, however, not affect the general positive link between confidence in emotion recognition and actual performance. Thus, while participants seemed to be able to calibrate their confidence ratings according to their recognition performance, a relative reduction in the scores might have occurred with higher social anxiety traits. This observation might be a reflection of self-related negative beliefs about one's own social skills which were likely formed in a public setting (Müller-Pinzler et al., 2019) and translated to a more global negative social skill assessment. Our study therefore provides evidence for an extension of previously described alterations in metacognitive beliefs to the domain of emotion recognition, which have been found to play a major role in the maintenance of social anxiety (Gkika et al., 2018). Further, as facial muscle responses to emotional expressions were not found to be altered depending on social anxiety traits in our sample, not only explicit emotion labeling but also implicit, automatic processes, i.e. facial mimicry, seem to be comparable across varying levels of social anxiety traits. While the link between corrugator responses and the recognition of sad and happy expression was not found to be modulated by social anxiety traits, the positive association between zygomaticus responses and the accurate labeling of happy expressions was weaker with higher social anxiety trait levels. This result, however, has to be interpreted with care given that emotion recognition performance for happy expression was at ceiling and there was mainly stronger variability in accuracies (i.e. also inaccurate responses) when the zygomaticus was not active in individuals with lower social anxiety traits. Further, the effect could not be reproduced in the analyses with relative accuracy as an outcome (see Supplemental material). Nevertheless, the function of smile mimicry might still be slightly shifted in 
social anxiety, from a tool to share others' affective states to a protection from negative social evaluation, as illustrated by an increased mimicry of polite smiles (Dijk et al., 2018).

\subsection{Modulations related to autistic traits}

In line with previous studies describing emotion recognition deficits in autism, we observed an overall reduced emotion recognition performance with higher autistic traits which became most apparent for fearful expressions (Frank et al., 2018; Sucksmith et al., 2013; Uljarevic \& Hamilton, 2013). The recognition of sad expressions, on the other hand, was not as strongly affected by autistic traits in the main analysis, and even improved with higher autistic trait levels in the relative accuracy analysis (see Supplemental Materials). This observation is compatible with a previous study which reported a better recognition of sadness expressions with higher autistic trait levels (Green \& Guo, 2018). Confidence in recognizing displays of sadness as well as fear was rated higher with higher autistic traits. Neutral and happy facial expressions, in contrast, received lower ratings with higher trait levels. The same pattern could be observed for the perceived emotional intensity ratings (see Supplemental Materials). Given the strong negative impact of autistic trait levels on recognition of fear displays, higher confidence and perceived intensity ratings seem particularly surprising here. The examination of the relationship between confidence and accuracy in emotion recognition, i.e. metacognitive sensitivity, revealed, however, that participants with higher autistic trait levels in our study were less able to scale their confidence according to their actual performance. Previous research on alterations in metacognitive judgments in autism has already described a complex pattern of both over- and underconfidence in the social-cognitive domain (DeBrabander et al., 2020). This pattern might depend on the distinct emotion category within an emotion recognition context which we could, however, not prove in our study. Thus, further research is needed to disentangle the complex pattern of metacognitive sensitivity in emotion recognition in autism. Our hypothesis that higher autistic trait level would result in reduced facial mimicry responses was also not confirmed. Even though we did not explicitly instruct participants to mimic, individuals with higher autistic trait levels still seemed to automatically show the expected facial muscle activation patterns, contradicting findings in clinical populations (McIntosh et al., 2006) as well as in healthy individuals with high autistic traits (Hermans 
et al., 2009). Importantly, it has been suggested that mimicry in ASD might especially be reduced for shorter presentation durations (Mathersul et al., 2013) and occur with a delay rather than not at all (Oberman et al., 2009), which we did not examine in our study. We did, however, observe a modulation in the link between facial mimicry and emotion recognition by autistic traits. In the recognition of sad expression, increased activity of the corrugator, i.e. mimicry, was less predictive of accurate recognition whereas the same applied to stronger zygomaticus activity in the recognition of happiness. While a sole evaluation of the latter effect would be difficult due to the ceiling performance in happiness recognition (see previous paragraph) as well as to a lack of reproducibility with a relative accuracy score (see Supplemental Material), the robust results concerning sadness recognition support the presence of a meaningful modulation. More specifically, mimicry-associated changes in facial muscle activity seem to be less integrated in explicit emotion processing, i.e. recognition, with higher autistic trait levels. This observation is in line with past research which did not find an effect of automatic, intentional or externally induced mimicry on reports of the participant's own emotion experience in individuals with ASD, while controls were considerably influenced (Stel et al., 2008). According to the idea of the existence of two routes in emotion recognition, a fast one involving proprioceptive (bottom-up) information and a long one involving knowledge-based (top-down) information (Stel \& van Knippenberg, 2008), the fast route might have been less employed in the recognition of sadness in participants with higher autistic traits. Since recognition performance of particularly sad expression was less impaired with higher autistic traits, judgments via the alternative, long route seemed to result in similarly successful judgments. Accordingly, while the development of emotion concepts might rely on embodiment (Winkielman et al., 2018), correct interpretations of observed affective states can be made without necessarily embodying other's emotions.

\subsection{General insights in facial mimicry and metacognition in emotion recognition}

In addition to the results specific to the trait dimensions, some general findings should receive attention. First, in line with previous research (Nummenmaa \& Calvo, 2015; Folz et al., in press), facial displays of happiness were by far the best recognized expression category. Surprised facial expressions were identified second-best, confirming claims that a salient mouth region, and 
specifically the presentation of teeth (daSilva et al., 2016), would yield a detection advantage (Calvo \& Nummenmaa, 2008). While neutral expressions were still recognized above the average, recognition performance for sad and especially fearful expressions was considerably worse, highlighting that these less frequently occurring expressions in everyday life might also be less familiar and more difficult to identify correctly (Calvo et al., 2014). Second, confidence in emotion recognition was found to be a significant predictor of recognition accuracy, indicating that our participants were correctly calibrating their metacognitive judgments according to their actual performance (e.g. Kelly \& Metcalfe, 2011). Human metacognition was described as a special cognitive ability which evolved through interacting and sharing experiences with others (Frith, 2012). In contexts in which no explicit feedback is available, such as our emotion recognition task but also daily social encounters, metacognitive judgments might provide relevant feedback. Third, similar to previous studies, we observed a consistent increase in muscle activity in the corrugator region if expressions of anger were viewed, and a decrease in corrugator activity as well as an increase in zygomaticus activity when happy facial expressions were displayed (e.g. Künecke et al., 2014; Rymarczyk et al., 2011, 2016a; Sato et al., 2008). Unexpectedly, displays of surprise were responded to with a similar, but considerably weaker, facial muscle response pattern as displays of happiness (i.e. decrease in corrugator activity and increase in zygomaticus activity). Given that some surprise expression were not of neutral valence but could rather be categorized as 'positively surprised', this positive affect might have been reflected in the facial EMG responses. Crucially, adding to the current discussion on the role of facial mimicry in emotion recognition (see Holland et al., 2020), our study could reveal a link between EMG responses to happy and sad expressions and associated recognition accuracy. More specifically, facial muscle activation patterns in the observer which are congruent to muscle activations underlying observed emotional facial expressions, i.e. activation of the zygomaticus and relaxation of the corrugator for happy expressions and activation of the corrugator for sad expressions, seem to go along with a better recognition performance. Sensorimotor simulation therefore indeed might be a relevant mechanism in understandings others' emotions (Wood et al., 2016). Given that the effects were not robust (see relative accuracy analysis in Supplemental Material) and we did not observe significant relationships 
for all expression categories (e.g. not for anger), an embodiment of observed emotional expressions does not seem necessary for a successful recognition (see also Folz et al, in press).

\subsection{Clinical implications}

From our findings suggesting that different components in emotion recognition might be altered in social anxiety and autism, disorder-specific clinical implications can be drawn. In line with theoretical models on the development and maintenance of social anxiety, as well as studies contrasting social performance and subjective evaluations in real-life scenarios (e.g. Dijk et al., 2009; Kashdan \& Savostyanova, 2011; Voncken \& Bögels, 2008), socially anxious individuals mainly seem to suffer from a cognitive bias, namely a generalized underconfidence in their social performance, which extends to the recognition of emotional facial expressions. This underconfidence might arise from a negatively biased post-even processing of social situations which has been more frequently observed and shown to be positively related to social anxiety (symptoms) in highly socially anxious individuals (Dannahy \& Stopa, 2007) as well as individuals with an SAD diagnosis (Helbig-Lang et al., 2016). Crucially, while being detrimental to social functioning, individuals with SAD have been found to hold more positive metacognitive beliefs about post-event processing. i.e. judging rumination as beneficial for their social skills (Gavric et al., 2017). Given the strong negative impact of cognitive biases in the maintenance of SAD, interventions focusing on overcoming those biases, like Metacognitive Training (MCT; Wells, 2009), seem most efficient in the treatment of SAD. While first empirical case studies confirm this suggestion by reporting general symptom reductions (Nordahl \& Wells, 2018), MCT might also yield promising outcomes with regard to increasing one's confidence in accurately understanding other's emotions. Emotion recognition alterations in autism, in contrast, seem to be qualitatively different. On the one hand, confidence in emotion recognition does not seem to be scaled to actual performance. Internal feedback, i.e. the“ feeling" how well one performed, might not be informative of actual performance in autism and, thus, cannot assist successful learning. Our findings suggest that, on the other hand, a simulation of observed expression might not be integrated to a similar degree in emotion processing in autism. This claim is supported by research showing a reduced access to bodily signals, i.e. interoceptive accuracy, next to a heightened sensitivity to those 
signals in autism (Garfinkel et al., 2016) which seems to be driven by comorbid alexithymia (Ketelaars et al., 2016; Shah et al., 2016). Consequently, while interventions targeting metacognitive abilities could help overcome the gap between actual performance and subjective judgments in individuals with ASD, a training focusing on the integration of information from the bodily component of an emotional experience could indirectly benefit emotion recognition.

\subsection{Limitations}

Despite our efforts to create a more naturalistic emotion recognition scenario by displaying spontaneous, dynamic facial expressions of emotion, participants still observed standardized stimuli in a controlled lab setting in our study. This limits the generalizability of our results as the interpretation of emotional expressions has been shown to be highly context-dependent (e.g. Hess \& Fischer, 2013; Israelashvili et al., 2019). More importantly, our study did not involve a real social context. Without an interaction partner who receives and responds to expressions from the participant, the social communicative function of emotional expressions, incl. a bidirectional coordination of affective states (Keltner \& Kring, 1998), may get lost (see also Schilbach et al., 2013). This limitation might also affect trait dimension-specific modulations in emotion perception. For example, in a real social situation, higher social anxiety levels have been associated with an enhanced mimicry of polite, but not enjoyment smiles (Dijk et al., 2018). Further, autism was argued to specifically become apparent in alterations in interpersonal dynamics, i.e. during bidirectional information exchange (Bolis et al., 2018; Peper et al., 2016). Consequently, future studies on emotion perception should be conducted in real social situations which allow for reciprocity and affect coordination. In addition to that, even though our observations on the impact of trait levels can give us hints with regard to alterations in clinical populations, we still collected data from a non-clinical sample. Once clinical symptoms which have a severe impact on an individual's life come into play, emotion processing might be altered differently, both qualitatively and quantitatively. For example, while sadness recognition was observed to be least impacted by autistic trait levels in our study, a reduced perceptual sensitivity has been specifically described for sad facial expressions in individuals with ASD (Wallace et al., 2011). This emotion-specific recognition impairment has been shown to extend to difficulties in interpreting 
sadness from animations which, in turn, has been related to worse daily social functioning in individuals with ASD (Boraston et al., 2007). Thus, in order to provide meaningful insights, results from studies including healthy participants with variations on clinical trait dimensions should always be confirmed in clinical populations as well as related to actual day-to-day experiences. Moreover, while our sample was not gender-balanced, gender differences in mimicry and its integration in emotion recognition have been reported in past research (Dimberg \& Lundquist, 1990; Stel \& van Knippenberg, 2008) and should therefore be considered more strongly in future studies. Lastly, as we did neither manipulate facial mimicry nor metacognition, our study does not allow for causality assumptions in their role in emotion recognition. A more fine-grained investigation of potential mediatory processes in the course of emotion perception and interpretation, such as processing of interoceptive information (Arnold et al., 2019; Critchley \& Garfinkel, 2017), might additionally benefit the understanding of functional and dysfunctional emotion processing and enable the formalization of testable theoretical models (see Smith et al., 2019).

\subsection{Conclusion}

In conclusion, our study provides evidence for distinct modulations of facial mimicry and metacognitive judgments in emotion recognition by autistic traits and social anxiety traits. Higher social anxiety traits were predominantly related to an underconfidence in emotion recognition, despite an unaltered performance, whereas higher autistic traits were associated with an overall worse recognition performance as well as a poorer calibration of performance judgments, and a less pronounced link between facial mimicry and emotion recognition. These trait dimension-specific patterns might also translate to the linked clinical disorders which, however, still has to be confirmed in future studies. In the long run, evidence-based interventions targeting alterations in specific components in the processing of other's emotions should provide more positive outcomes in social functioning and thereby improve the quality of life in individuals with SAD and individuals with ASD. 


\section{References}

Altman, D. G., \& Royston, P. (2006). The cost of dichotomising continuous variables. British Medical Journal, 332(7549), 1080. https://doi.org/10.1136/bmj.332.7549.1080

Alves, N. T. (2013). Recognition of static and dynamic facial expressions: A study review. Estudos de Psicologia, 18(1), 125-130. https://doi.org/10.1590/S1413-294X2013000100020

Ambadar, Z., Schooler, J. W., \& Cohn, J. F. (2005). Deciphering the Enigmatic Face. Society, 16(5), 403-410. https://doi.org/10.1111/j.0956-7976.2005.01548.x

Amin, N., Foa, E. B., \& Coles, M. E. (1998). Negative interpretation bias in social phobia. Behaviour Research and Therapy, 36(10), 945-957. https://doi.org/10.1016/S0005-7967(98)00060-6

Arnold, A. J., Winkielman, P., \& Dobkins, K. (2019). Interoception and Social Connection. Frontiers in Psychology, 10, 1-6. https://doi.org/10.3389/fpsyg.2019.02589

Arrais, K. C., Machado-de-Sousa, J. P., Trzesniak, C., Filho, A. S., Ferrari, M. C. F., Osório, F. L., Loureiro, S. R., Nardi, A. E., Hetem, L. A. B., Zuardi, A. W., Hallak, J. E. C., \& Crippa, J. A. S. (2010). Social anxiety disorder women easily recognize fearfull, sad and happy faces: The influence of gender. Journal of Psychiatric Research, 44(8), 535-540. https://doi.org/10.1016/j.jpsychires.2009.11.003

Baron-Cohen, S., Wheelwright, S., Skinner, R., Martin, J., \& Clubley, E. (2001). The Autism Spectrum Quotient (AQ): Evidence from Asperger Syndrome/High-Functioning Autism, Males and Females, Scientists and Mathematicians. Journal of Autism and Developmental Disorders, 31(1), 5-17. https://doi.org/10.1023/A:1005653411471

Bègue, I., Vaessen, M., Hofmeister, J., Pereira, M., Schwartz, S., \& Vuilleumier, P. (2019). Confidence of emotion expression recognition recruits brain regions outside the face perception network. November 2018, 81-95. https://doi.org/10.1093/scan/nsy102

Bogart, K. R., \& Matsumoto, D. (2010). Facial mimicry is not necessary to recognize emotion: Facial expression recognition by people with Moebius syndrome. Social Neuroscience, 5(2), 241-251. https://doi.org/10.1080/17470910903395692

Bolis, D., Balsters, J., Wenderoth, N., Becchio, C., \& Schilbach, L. (2018). Beyond Autism: Introducing the Dialectical Misattunement Hypothesis and a Bayesian Account of Intersubjectivity. Psychopathology, 50(6), 355-372. https://doi.org/10.1159/000484353 
Boraston, Z., Blakemore, S. J., Chilvers, R., \& Skuse, D. (2007). Impaired sadness recognition is linked to social interaction deficit in autism. Neuropsychologia, 45(7), 1501-1510. https://doi.org/10.1016/j.neuropsychologia.2006.11.010

Boraston, Z., Corden, B., Miles, L. K., Skuse, D. H., \& Blakemore, S. J. (2008). Brief report: Perception of genuine and posed smiles by individuals with autism. Journal of Autism and Developmental Disorders, 38(3), 574-580. https://doi.org/10.1007/s10803-007-0421-1

Brown, S. L., \& Schwartz, G. E. (1980). Relationships between facial electromyography and subjective experience during affective imagery. Biological Psychology, 11(1), 49-62. https://doi.org/10.1016/0301-0511(80)90026-5

Buck, R. (1980). Nonverbal behavior and the theory of emotion: The facial feedback hypothesis. Journal of Personality and Social Psychology, 38(5), 811-824. https://doi.org/10.1037/00223514.38.5.811

Bui, E., Anderson, E., Goetter, E. M., Campbell, A. A., Fischer, L. E., Barrett, L. F., \& Simon, N. M. (2017). Heightened sensitivity to emotional expressions in generalised anxiety disorder, compared to social anxiety disorder, and controls. Cognition and Emotion, 31(1), 119-126. https://doi.org/10.1080/02699931.2015.1087973

Calvo, M. G., Gutiérrez-García, A., Fernández-Martín, A., \& Nummenmaa, L. (2014). Recognition of Facial Expressions of Emotion is Related to their Frequency in Everyday Life. Journal of Nonverbal Behavior, 38(4), 549-567. https://doi.org/10.1007/s10919-014-0191-3

Calvo, M. G., \& Nummenmaa, L. (2008). Detection of Emotional Faces: Salient Physical Features Guide Effective Visual Search. Journal of Experimental Psychology: General, 137(3), 471-494. https://doi.org/10.1037/a0012771

Clark, D. M., \& Wells, A. (1995). A cognitive model of social phobia. In R. G. Heimberg, M. Liebowitz, D. Hope, \& F. Schneier (Eds.), Social phobia: Diagnosis, assessment, and treatment (pp. 69-93). New York Guilford Press.

Cohn, J. F. (2007). Foundations of human computing: Facial expression and emotion. Lecture Notes in Computer Science (Including Subseries Lecture Notes in Artificial Intelligence and Lecture Notes in Bioinformatics), 4451 LNAI, 1-16. https://doi.org/10.1007/978-3-540-72348-6_1

Critchley, H. D., \& Garfinkel, S. N. (2017). Interoception and emotion. Current Opinion in Psychology, 17, 7-14. https://doi.org/10.1016/j.copsyc.2017.04.020 
Damasio, A. (1996). The somatic marker hypothesis and the possible functions of the prefrontal cortex. Philosophical Transactions of the Royal Society of London. Series B, 351, 1413-1420. https://doi.org/10.1098/rstb.1996.0125

Dannahy, L., \& Stopa, L. (2007). Post-event processing in social anxiety. Behaviour Research and Therapy, 45(6), 1207-1219. https://doi.org/10.1016/j.brat.2006.08.017

daSilva, E. B., Crager, K., Geisler, D., Newbern, P., Orem, B., \& Puce, A. (2016). Something to sink your teeth into: The presence of teeth augments ERPs to mouth expressions. NeuroImage, 127, 227-241. https://doi.org/10.1016/j.neuroimage.2015.12.020

Davies, H., Wolz, I., Leppanen, J., Fernandez-Aranda, F., Schmidt, U., \& Tchanturia, K. (2016). Facial expression to emotional stimuli in non-psychotic disorders: A systematic review and metaanalysis. Neuroscience and Biobehavioral Reviews, 64, 252-271. https://doi.org/10.1016/j.neubiorev.2016.02.015

DeBrabander, K. M., Pinkham, A. E., Ackerman, R. A., Jones, D. R., \& Sasson, N. J. (2020). Cognitive and Social Cognitive Self - assessment in Autistic Adults. Journal of Autism and Developmental Disorders, 51(7), 2354-2368. https://doi.org/10.1007/s10803-020-04722-x

Dentakos, S., Saoud, W., Ackerman, R., \& Toplak, M. E. (2019). Does domain matter? Monitoring accuracy across domains. Metacognition and Learning, 14(3), 413-436. https://doi.org/10.1007/s11409-019-09198-4

Dignath, D., Berger, A., Spruit, I. M., \& van Steenbergen, H. (2019). Temporal dynamics of errorrelated corrugator supercilii and zygomaticus major activity: Evidence for implicit emotion regulation following errors. International Journal of Psychophysiology, 146(August), 208-216. https://doi.org/10.1016/j.jpsycho.2019.10.003

Dijk, C., Fischer, A. H., Morina, N., Eeuwijk, C. Van, \& Kleef, G. A. Van. (2018). Effects of Social Anxiety on Emotional Mimicry and Contagion: Feeling Negative, but Smiling Politely. Journal of Nonverbal Behavior, 42(1), 81-99. https://doi.org/10.1007/s10919-017-0266-z

Dijk, C., Voncken, M. J., \& de Jong, P. J. (2009). I blush, therefore I will be judged negatively: influence of false blush feedback on anticipated others' judgments and facial coloration in high and low blushing-fearfuls. Behaviour Research and Therapy, 47(7), 541-547. https://doi.org/10.1016/j.brat.2009.03.005

Dimberg, U. (1982). Facial Reactions to Facial Expressions. Psychophysiology, 19(6), 643-647. https://doi.org/10.1111/j.1469-8986.1982.tb02516.x 
Dimberg, U. (1997). Social fear and expressive reactions to social stimuli. Scandinavian Journal of Psychology, 38(3), 171-174. https://doi.org/10.1111/1467-9450.00024

Dimberg, U., \& Lundquist, L. O. (1990). Gender differences in facial reactions to facial expressions. Biological Psychology, 30(2), 151-159. https://doi.org/10.1016/0301-0511(90)90024-Q

Dimberg, U., \& Thunberg, M. (2007). Speech anxiety and rapid emotional reactions to angry and happy facial expressions: Personality and Social Sciences. Scandinavian Journal of Psychology, 48(4), 321-328. https://doi.org/10.1111/j.1467-9450.2007.00586.x

Dobs, K., Bülthoff, I., \& Schultz, J. (2018). Use and usefulness of dynamic face stimuli for face perception studies-a review of behavioral findings and methodology. Frontiers in Psychology, 9(AUG), 1-7. https://doi.org/10.3389/fpsyg.2018.01355

Drimalla, H., Landwehr, N., Hess, U., \& Dziobek, I. (2019). From face to face: the contribution of facial mimicry to cognitive and emotional empathy. Cognition and Emotion, 33(8), 1672-1686. https://doi.org/10.1080/02699931.2019.1596068

Ekman, P., Levenson, R. W., \& Friesen, W. V. (1983). Autonomic Nervous System Activity Distinguishes Among Emotions. Science, 221(4616), 1208-1210.

Ekman, P., \& Rosenberg, E. (2005). What the Face Reveals: Basic and Applied Studies of Spontaneous Expression Using the Facial Action Coding System (FACS). In What the Face Reveals. Oxford University Press.

Enticott, P. G., Kennedy, H. A., Johnston, P. J., Rinehart, N. J., Tonge, B. J., Taffe, J. R., \& Fitzgerald, P. B. (2014). Emotion recognition of static and dynamic faces in autism spectrum disorder. Cognition and Emotion, 28(6), 1110-1118. https://doi.org/10.1080/02699931.2013.867832

Fleming, S. M., \& Lau, H. C. (2014). How to measure metacognition. Frontiers in Human Neuroscience, 8(July), 1-9. https://doi.org/10.3389/fnhum.2014.00443

Frank, R., Schulze, L., Hellweg, R., Koehne, S., \& Roepke, S. (2018). Impaired detection and differentiation of briefly presented facial emotions in adults with high-functioning autism and asperger syndrome. Behaviour Research and Therapy, 104(June 2017), 7-13. https://doi.org/10.1016/j.brat.2018.02.005

Fridlund, A. J., \& Cacioppo, J. T. (1986). Guidelines for Human Electromyographic Research. Psychophysiology, 23(5), 567-589. https://doi.org/10.1111/j.1469-8986.1986.tb00676.x 
Frith, C. (2009). Role of facial expressions in social interactions. Philosophical Transactions of the Royal Society B: Biological Sciences, 364(1535), 3453-3458. https://doi.org/10.1098/rstb.2009.0142

Frith, C. (2012). The role of metacognition in human social interactions. Philosophical Transactions of the Royal Society B: Biological Sciences, 367(1599), 2213-2223. https://doi.org/10.1098/rstb.2012.0123

Garfinkel, S. N., Tiley, C., O’Keeffe, S., Harrison, N. A., Seth, A. K., \& Critchley, H. D. (2016). Discrepancies between dimensions of interoception in autism: Implications for emotion and anxiety. Biological Psychology, 114, 117-126. https://doi.org/10.1016/j.biopsycho.2015.12.003

Gavric, D., Moscovitch, D. A., Rowa, K., \& McCabe, R. E. (2017). Post-event processing in social anxiety disorder: Examining the mediating roles of positive metacognitive beliefs and perceptions of performance. Behaviour Research and Therapy, 91, 1-12. https://doi.org/10.1016/j.brat.2017.01.002

Gilboa-Schechtman, E., \& Shachar-Lavie, I. (2013). More than a face: a unified theoretical perspective on nonverbal social cue processing in social anxiety. Frontiers in Human Neuroscience, 7(December), 1-14. https://doi.org/10.3389/fnhum.2013.00904

Gkika, S., Wittkowski, A., \& Wells, A. (2018). Social cognition and metacognition in social anxiety: A systematic review. Clinical Psychology and Psychotherapy, 25(1), 10-30. https://doi.org/10.1002/cpp.2127

Goodman, L. R., Corkum, P., \& Johnson, S. A. (2017). A metacognitive training pilot study for adolescents with autism spectrum disorder: Lessons learned from the preliminary stages of intervention development. Journal of Intellectual and Developmental Disability, 42(2), 204-210. https://doi.org/10.3109/13668250.2016.1210104

Green, C., \& Guo, K. (2018). Factors contributing to individual differences in facial expression categorisation. Cognition and Emotion, 32(1), 37-48. https://doi.org/10.1080/02699931.2016.1273200

Gutiérrez-García, A., \& Calvo, M. G. (2016). Social anxiety and trustworthiness judgments of dynamic facial expressions of emotion. Journal of Behavior Therapy and Experimental Psychiatry, 52, 119-127. https://doi.org/10.1016/j.jbtep.2016.04.003 
Gutiérrez-García, A., \& Calvo, M. G. (2017). Social anxiety and threat-related interpretation of dynamic facial expressions: Sensitivity and response bias. Personality and Individual Differences, 107, 10-16. https://doi.org/10.1016/j.paid.2016.11.025

Hartman, L. M. (1983). A metacognitive model of social anxiety: Implications for treatment. Clinical Psychology Review, 3(4), 435-456. https://doi.org/10.1016/0272-7358(83)90023-5

Hatfield, E., Cacioppo, J. T., \& Rapson, R. L. (1993). Emotional contagion. Current Directions in Psychological Science, 2(3), 96-99. https://doi.org/10.1111/1467-8721.ep10770953

Helbig-Lang, S., von Auer, M., Neubauer, K., Murray, E., \& Gerlach, A. L. (2016). Post-event processing in social anxiety disorder after real-life social situations - An ambulatory assessment study. Behaviour Research and Therapy, 84, 27-34. https://doi.org/10.1016/j.brat.2016.07.003

Hermans, E. J., van Wingen, G., Bos, P. A., Putman, P., \& van Honk, J. (2009). Reduced spontaneous facial mimicry in women with autistic traits. Biological Psychology, 80(3), 348-353. https://doi.org/10.1016/j.biopsycho.2008.12.002

Hess, U., \& Blairy, S. (2001). Facial mimicry and emotional contagion to dynamic emotional facial expressions and their influence on decoding accuracy. International Journal of Psychophysiology, 40(2), 129-141. https://doi.org/10.1016/S0167-8760(00)00161-6

Hess, U., \& Fischer, A. (2013). Emotional Mimicry as Social Regulation. Personality and Social Psychology Review, 17(2), 142-157. https://doi.org/10.1177/1088868312472607

Hirsch, C. R., \& Clark, D. M. (2004). Information-processing bias in social phobia. Clinical Psychology Review, 24(7), 799-825. https://doi.org/10.1016/j.cpr.2004.07.005

Holland, A. C., O’Connell, G., \& Dziobek, I. (2020). Facial mimicry, empathy, and emotion recognition: a meta-analysis of correlations. Cognition and Emotion, 1-19. https://doi.org/10.1080/02699931.2020.1815655

Hothorn T, Bretz F, and Westfall P (2008). Simultaneous Inference in General Parametric Models. Biometrical Journal 50(3), 346--363.

Hunter, L. R., Buckner, J. D., \& Schimdt, N. B. (2009). Interpreting facial expressions: The influence of social anxiety, emotional valence, and race. Journal of Anxiety Disorders, 23(1), 1-7. https://doi.org/10.1016/j.janxdis.2008.10.004.Interpreting 
Israelashvili, J., Aviezer, H., \& Hassin, R. (2019). When Emotions Run High: A Critical Role for Context in the Unfolding of Dynamic, Real-Life Facial Affect. Emotion, 19(3), 558-562. https://doi.org/10.1037/emo0000441

James, W. (1884). What is an Emotion? Mind, 9, 188-205. http://mind.oxfordjournals.org/content/osIX/34/188

Joormann, J., \& Gotlib, I. H. (2006). Is this happiness i see? Biases in the identification of emotional facial expressions in depression and social phobia. Journal of Abnormal Psychology, 115(4), 705-714. https://doi.org/10.1037/0021-843X.115.4.705

Kashdan, T. B., \& Savostyanova, A. A. (2011). Capturing the Biases of Socially Anxious People by Addressing Partner Effects and Situational Parameters. Behavior Therapy, 42(2), 211-233. https://doi.org/10.1016/j.earlhumdev.2006.05.022

Keating, C. T., Fraser, D. S., Sowden, S., \& Cook, J. L. (2021). Differences Between Autistic and Non-Autistic Adults in the Recognition of Anger from Facial Motion Remain after Controlling for Alexithymia. Journal of Autism and Developmental Disorders, 0123456789. https://doi.org/10.1007/s10803-021-05083-9

Kelly, K. J., \& Metcalfe, J. (2011). Metacognition of Emotional Face Recognition. Emotion, 11(4), 896-906. https://doi.org/10.1037/a0023746

Keltner, D., \& Kring, A. M. (1998). Emotion, social function, and psychopathology. Review of General Psychology, 2(3), 320-342. https://doi.org/10.1037/1089-2680.2.3.320

Ketelaars, M. P., In'T Velt, A., Mol, A., Swaab, H., \& Van Rijn, S. (2016). Emotion recognition and alexithymia in high functioning females with autism spectrum disorder. Research in Autism Spectrum Disorders, 21, 51-60. https://doi.org/10.1016/j.rasd.2015.09.006

Kret, M. E. (2015). Emotional expressions beyond facial muscle actions . A call for studying autonomic signals and their impact on social perception. Frontiers in Psychology, 6, 1-10. https://doi.org/10.3389/fpsyg.2015.00711

Kret, M. E., Prochazkova, E., Sterck, E. H. M., \& Clay, Z. (2020). Emotional expressions in human and non-human great apes. Neuroscience \& Biobehavioral Reviews. https://doi.org/10.1016/j.neubiorev.2020.01.027

Krumhuber, E. G., Kappas, A., \& Manstead, A. S. R. (2013). Effects of dynamic aspects of facial expressions: A review. Emotion Review, 5(1), 41-46. https://doi.org/10.1177/1754073912451349 
Krumhuber, E. G., Küster, D., Namba, S., Shah, D., \& Calvo, M. G. (2019). Emotion Recognition From Posed and Spontaneous Dynamic Expressions: Human Observers Versus Machine Analysis. Emotion. https://doi.org/10.1037/emo0000712

Krumhuber, E. G., Skora, L., Küster, D., \& Fou, L. (2017). A Review of Dynamic Datasets for Facial Expression Research. Emotion Review, 9(3), 280-292. https://doi.org/10.1177/1754073916670022

Künecke, J., Hildebrandt, A., Recio, G., Sommer, W., \& Wilhelm, O. (2014). Facial EMG responses to emotional expressions are related to emotion perception ability. PLoS ONE, 9(1). https://doi.org/10.1371/journal.pone.0084053

Kuznetsova A, Brockhoff PB, Christensen RHB (2017). "ImerTest Package: Tests in Linear Mixed Effects Models.”_Journal of Statistical Software_, *82*(13), 1-26. doi: 10.18637/jss.v082.i13 (URL: https://doi.org/10.18637/jss.v082.i13).

Locke, K. D., \& Mitchell, G. E. (2016). Self-perceptions, parent-perceptions, and meta-perceptions of the interpersonal efficacy of adolescents with autism spectrum disorder. Research in Autism Spectrum Disorders, 31, 19-29. https://doi.org/10.1016/j.rasd.2016.07.006

Lüdecke D (2021)._sjPlot: Data Visualization for Statistics in Socia Science_. R package version 2.8.9, <URL: https://CRAN.R-project.org/package=sjPlot $>$.

Machado-de-Sousa, J. P., Arrais, K. C., Alves, N. T., Chagas, M. H. N., de Meneses-Gaya, C., Crippa, J. A. de S., \& Hallak, J. E. C. (2010). Facial affect processing in social anxiety: Tasks and stimuli. Journal of Neuroscience Methods, 193(1), 1-6. https://doi.org/10.1016/j.jneumeth.2010.08.013

Mathersul, D., McDonald, S., \& Rushby, J. A. (2013). Automatic facial responses to briefly presented emotional stimuli in autism spectrum disorder. Biological Psychology, 94(2), 397-407. https://doi.org/10.1016/j.biopsycho.2013.08.004

McIntosh, D. N., Reichmann-Decker, A., Winkielman, P., \& Wilbarger, J. L. (2006). When the social mirror breaks: Deficits in automatic, but not voluntary, mimicry of emotional facial expressions in autism. Developmental Science, 9(3), 295-302. https://doi.org/10.1111/j.14677687.2006.00492.x 
Mennin, D. S., Fresco, D. M., Heimberg, R. G., Schneier, F. R., Davies, S. O., \& Liebowitz, M. R. (2002). Screening for social anxiety disorder in the clinical setting: Using the Liebowitz Social Anxiety Scale. Journal of Anxiety Disorders, 16(6), 661-673. https://doi.org/10.1016/S08876185(02)00134-2

Montagne, B., Schutters, S., Westenberg, H. G. M., Van Honk, J., Kessels, R. P. C., \& De Haan, E. H. F. (2006). Reduced sensitivity in the recognition of anger and disgust in social anxiety disorder. Cognitive Neuropsychiatry, 11(4), 389-401. https://doi.org/10.1080/13546800444000254

Müller-Pinzler, L., Czekalla, N., Mayer, A. V., Stolz, D. S., Gazzola, V., Keysers, C., Paulus, F. M., \& Krach, S. (2019). Negativity-bias in forming beliefs about own abilities. Scientific Reports, 9(1), 1-15. https://doi.org/10.1038/s41598-019-50821-w

Neal, D. T., \& Chartrand, T. L. (2011). Embodied emotion perception: Amplifying and dampening facial feedback modulates emotion perception accuracy. Social Psychological and Personality Science, 2(6), 673-678. https://doi.org/10.1177/1948550611406138

Niedenthal, P. M. (2007). Embodying Emotion. Science, 316(5827), 1002-1006. https://doi.org/10.1126/science.1136930

Noldus. (2014). FaceReader: Tool for automatic analysis of facial expression: Version 6.0. Noldus Information Technology B.V

Nordahl, H., Ødegaard, I. H., Hjemdal, O., \& Wells, A. (2019). A test of the goodness of fit of the generic metacognitive model of psychopathology symptoms. BMC Psychiatry, 19(1), 1-9. https://doi.org/10.1186/s12888-019-2266-5

Nordahl, H., \& Wells, A. (2018). Metacognitive therapy for social anxiety disorder: An A-B replication series across social anxiety subtypes. Frontiers in Psychology, 9(APR), 1-7. https://doi.org/10.3389/fpsyg.2018.00540

Nummenmaa, L., \& Calvo, M. G. (2015). Supplemental Material for Dissociation Between Recognition and Detection Advantage for Facial Expressions: A Meta-Analysis. Emotion, 15(1), 1-14. https://doi.org/10.1037/emo0000042.supp

Oberman, L. M., Winkielman, P., \& Ramachandran, V. S. (2007). Face to face: Blocking facial mimicry can selectively impair recognition of emotional expressions. Social Neuroscience, 2(34), 167-178. https://doi.org/10.1080/17470910701391943 
Oberman, L. M., Winkielman, P., \& Ramachandran, V. S. (2009). Slow echo: Facial EMG evidence for the delay of spontaneous, but not voluntary, emotional mimicry in children with autism spectrum disorders. Developmental Science, 12(4), 510-520. https://doi.org/10.1111/j.14677687.2008.00796.x

Olszanowski, M., Wróbel, M., \& Hess, U. (2020). Mimicking and sharing emotions: a re-examination of the link between facial mimicry and emotional contagion. Cognition and Emotion, 34(2), 367376. https://doi.org/10.1080/02699931.2019.1611543

Peper, C. (Lieke)E, van der Wal, S. J., \& Begeer, S. (2016). Autism in action: Reduced bodily connectedness during social interactions? Frontiers in Psychology, 7(NOV), 1-6. https://doi.org/10.3389/fpsyg.2016.01862

Peter-Ruf, C., Kirmse, U., Pfeiffer, S., Schmidt, M., Wilhelm, F. H., \& In-Albon, T. (2017). Emotion Regulation in High and Low Socially Anxious Individuals : An Experimental Study Investigating Emotional Mimicry, Emotion Recognition, and Self-Reported Emotion Regulation. Journal of Depression and Anxiety Disorders, 1(1), 17-26.

Philip, R. C. M., Whalley, H. C., Stanfield, A. C., Sprengelmeyer, R., Santos, I. M., Young, A. W., Atkinson, A. P., Calder, A. J., Johnstone, E. C., Lawrie, S. M., \& Hall, J. (2010). Deficits in facial, body movement and vocal emotional processing in autism spectrum disorders. Psychological Medicine, 40(11), 1919-1929. https://doi.org/10.1017/S0033291709992364

Prochazkova, E., \& Kret, M. E. (2017). Connecting minds and sharing emotions through mimicry : A neurocognitive model of emotional contagion. Neuroscience and Biobehavioral Reviews, 80, 99 114. https://doi.org/10.1016/j.neubiorev.2017.05.013

Psychology Software Tools, Inc. [E-Prime 3.0]. (2016). Psychology Software Tools. https://www.pstnet.com.

R Core Team (2020). R: A language and environment for statistical computing. R Foundation for Statistical Computing, Vienna, Austria. https://www.R-project.org/.

Rapee, R. M., \& Heimberg, R. G. (1997). A cognitive-behavioral model of anxiety in social phobia. Behaviour Research and Therapy, 35(8), 741-756. https://doi.org/10.1016/S00057967(97)00022-3

Richards, A., French, C. C., Calder, A. J., Webb, B., Fox, R., \& Young, A. W. (2002). AnxietyRelated Bias in the Classification of Emotionally Ambiguous Facial Expressions. Emotion, 2(3), 273-287. https://doi.org/10.1037/1528-3542.2.3.273 
Rouault, M., Seow, T., Gillan, C. M., \& Fleming, S. M. (2018). Psychiatric Symptom Dimensions Are Associated With Dissociable Shifts in Metacognition but Not Task Performance. Biological Psychiatry, 84(6), 443-451. https://doi.org/10.1016/j.biopsych.2017.12.017

Rozga, A., King, T. Z., Vuduc, R. W., \& Robins, D. L. (2013). Undifferentiated facial electromyography responses to dynamic, audio-visual emotion displays in individuals with autism spectrum disorders. Developmental Science, 16(4), 499-514.

https://doi.org/10.1111/desc.12062

Rymarczyk, K., Biele, C., Grabowska, A., \& Majczynski, H. (2011). EMG activity in response to static and dynamic facial expressions. International Journal of Psychophysiology, 79(2), 330333. https://doi.org/10.1016/j.ijpsycho.2010.11.001

Rymarczyk, K., Zurawski, Ł., Jankowiak-Siuda, K., \& Szatkowska, I. (2016). Do dynamic compared to static facial expressions of happiness and anger reveal enhanced facial mimicry? PLoS ONE, 11(7), 1-15. https://doi.org/10.1371/journal.pone.0158534

Rytwinski, N. K., Fresco, D. M., Heimberg, R. G., Coles, M. E., Liebowitz, M. R., Cissell, S., Stein, M. B., \& Hofmann, S. G. (2009). Screening for social anxiety disorder with the self-report version of the liebowitz social anxiety scale. Depression and Anxiety, 26(1), 34-38. https://doi.org/10.1002/da.20503

Sato, W., Fujimura, T., \& Suzuki, N. (2008). Enhanced facial EMG activity in response to dynamic facial expressions. International Journal of Psychophysiology, 70(1), 70-74. https://doi.org/10.1016/j.ijpsycho.2008.06.001

Sauter, D. A., \& Fischer, A. H. (2018). Can perceivers recognise emotions from spontaneous expressions? Cognition and Emotion, 32(3), 504-515. https://doi.org/10.1080/02699931.2017.1320978

Sawyer, A. C. P., Williamson, P., \& Young, R. (2014). Metacognitive processes in emotion recognition: Are they different in adults with Asperger's disorder? Journal of Autism and Developmental Disorders, 44(6), 1373-1382. https://doi.org/10.1007/s10803-013-1999-0

Schilbach, L., Timmermans, B., Reddy, V., Costall, A., Bente, G., Schlicht, T., \& Vogeley, K. (2013). Toward a second-person neuroscience. Behavioral and Brain Sciences, 36(4), 393-414. https://doi.org/10.1017/S0140525X12000660

Schultz, J., \& Pilz, K. S. (2009). Natural facial motion enhances cortical responses to faces. Experimental Brain Research, 194(3), 465-475. https://doi.org/10.1007/s00221-009-1721-9 
Shah, P., Hall, R., Catmur, C., \& Bird, G. (2016). Alexithymia, not autism, is associated with impaired interoception. Cortex, 81, 215-220. https://doi.org/10.1016/j.cortex.2016.03.021

Smith, R., Parr, T., \& Friston, K. J. (2019). Simulating Emotions: An Active Inference Model of Emotional State Inference and Emotion Concept Learning. Frontiers in Psychology, 10(December), 1-24. https://doi.org/10.3389/fpsyg.2019.02844

Sonkusare, S., Breakspear, M., \& Guo, C. (2019). Naturalistic Stimuli in Neuroscience: Critically Acclaimed. Trends in Cognitive Sciences, 23(8), 699-714. https://doi.org/10.1016/j.tics.2019.05.004

Staugaard, S. R. (2010). Threatening faces and social anxiety: A literature review. Clinical Psychology Review, 30(6), 669-690. https://doi.org/10.1016/j.cpr.2010.05.001

Stel, M., van den Heuvel, C., \& Smeets, R. C. (2008). Facial Feedback Mechanisms in Autistic Spectrum Disorders. Journal of Autism and Developmental Disorders, 38, 1250-1258. https://doi.org/10.1007/s10803-007-0505-y

Stel, M., \& van Knippenberg, A. (2008). The Role of Facial Mimicry in the Recognition of Affect. Psychological Science, 19(10), 984-986.

Sucksmith, E., Allison, C., Baron-Cohen, S., Chakrabarti, B., \& Hoekstra, R. A. (2013). Empathy and emotion recognition in people with autism, first-degree relatives, and controls. Neuropsychologia, 51(1), 98-105. https://doi.org/10.1016/j.neuropsychologia.2012.11.013

Torro-Alves, N., Bezerra, I. A. de O., e Claudino, R. G., Rodrigues, M. R., Machado-de-Sousa, J. P., Osório, F. de L., \& Crippa, J. A. (2016). Facial emotion recognition in social anxiety: The influence of dynamic information. Psychology and Neuroscience, 9(1), 1-11. https://doi.org/10.1037/pne0000042

Trevisan, D. A., \& Birmingham, E. (2016). Are emotion recognition abilities related to everyday social functioning in ASD? A meta-analysis. Research in Autism Spectrum Disorders, 32, 24-42. https://doi.org/10.1016/j.rasd.2016.08.004

Uljarevic, M., \& Hamilton, A. (2013). Recognition of emotions in autism: A formal meta-analysis. Journal of Autism and Developmental Disorders, 43(7), 1517-1526. https://doi.org/10.1007/s10803-012-1695-5 
Voncken, M. J., \& Bögels, S. M. (2008). Social performance deficits in social anxiety disorder: Reality during conversation and biased perception during speech. Journal of Anxiety Disorders, 22(8), 1384-1392. https://doi.org/10.1016/j.janxdis.2008.02.001

Vrana, S. R., \& Gross, D. (2004). Reactions to facial expressions: Effects of social context and speech anxiety on responses to neutral, anger, and joy expressions. Biological Psychology, 66(1), 63-78. https://doi.org/10.1016/j.biopsycho.2003.07.004

Wallhoff, F., Schuller, B., Hawellek, M., \& Rigoll, G. (2006). Efficient Recognition of Authentic Dynamic Facial Expressions on the Feedtum Database. 2006 IEEE International Conference on Multimedia and Expo. https://doi.org/10.1109/icme.2006.262433

Wang, S., \& Adolphs, R. (2017). Reduced specificity in emotion judgment in people with autism spectrum disorder. Neuropsychologia, 99(March), 286-295. https://doi.org/10.1016/j.neuropsychologia.2017.03.024

Weiss, E., Siedentopf, C. M., Hofer, A., Deisenhammer, E. A., Hoptman, M. J., Kremser, C., Golaszewski, S., Felber, S., Fleischhacker, W. W., \& Delazer, M. (2003). Sex differences in brain activation pattern during a visuospatial cognitive task: A functional magnetic resonance imaging study in healthy volunteers. Neuroscience Letters, 344(3), 169-172. https://doi.org/10.1016/S0304-3940(03)00406-3

Wells, A. (2009). Metacognitive therapy for anxiety and depression. Guilford.

Westfall, J. (2015). PANGEA: Power ANalysis for GEneral Anova designs. Unpublished manuscript. Available at http://jakewestfall.org/publications/pangea.pdf

Winkielman, P., Coulson, S., \& Niedenthal, P. (2018). Dynamic grounding of emotion concepts. Philosophical Transactions of the Royal Society B: Biological Sciences, 373(1752). https://doi.org/10.1098/rstb.2017.0127

Wood, A., Rychlowska, M., Korb, S., \& Niedenthal, P. (2016). Fashioning the Face: Sensorimotor Simulation Contributes to Facial Expression Recognition. Trends in Cognitive Sciences, 20(3), 227-240. https://doi.org/10.1016/j.tics.2015.12.010

Zwick, J. C., \& Wolkenstein, L. (2017). Facial emotion recognition, theory of mind and the role of facial mimicry in depression. Journal of Affective Disorders, 210, 90-99. https://doi.org/10.1016/j.jad.2016.12.022 